\title{
A normalized basis for quintic Powell-Sabin splines
}

\author{
Hendrik Speleers
}

Report TW556, January 2010

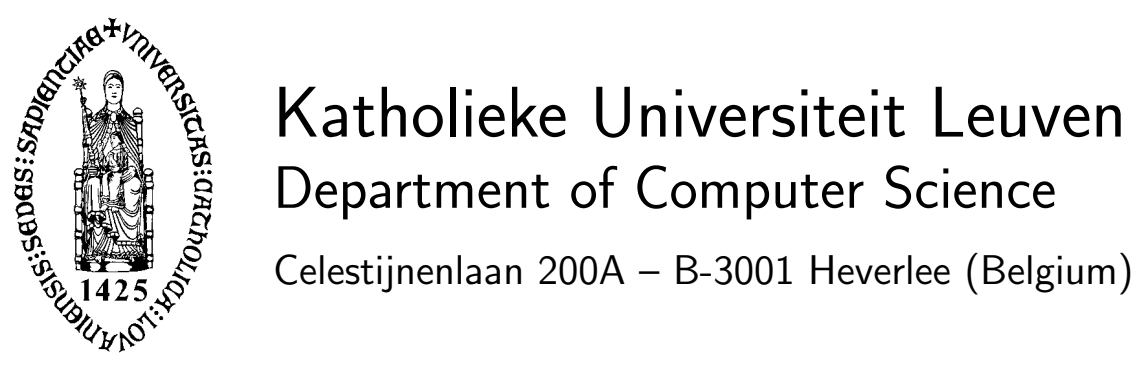




\title{
A normalized basis for quintic Powell-Sabin splines
}

\author{
Hendrik Speleers
}

Report TW 556, January 2010

Department of Computer Science, K.U.Leuven

\begin{abstract}
We construct a suitable normalized B-spline representation for $C^{2}$-continuous quintic Powell-Sabin splines. The basis functions have a local support, they are nonnegative, and they form a partition of unity. The construction is based on the determination of a set of triangles that must contain a specific set of points. We are able to define control points and cubic control polynomials which are tangent to the spline surface. We also show how to compute the Bézier control net of such a spline in a stable way.
\end{abstract}

Keywords : quintic Powell-Sabin splines, normalized B-splines, control points, control polynomials, Bézier control net

MSC : Primary : 65D07, Secondary : 65D17, 68U07 


\title{
A normalized basis for quintic Powell-Sabin splines
}

\author{
Hendrik Speleers \\ Department of Computer Science, Katholieke Universiteit Leuven \\ Celestijnenlaan 200A, B-3001 Leuven, Belgium
}

\begin{abstract}
We construct a suitable normalized B-spline representation for $C^{2}$-continuous quintic PowellSabin splines. The basis functions have a local support, they are nonnegative, and they form a partition of unity. The construction is based on the determination of a set of triangles that must contain a specific set of points. We are able to define control points and cubic control polynomials which are tangent to the spline surface. We also show how to compute the Bézier control net of such a spline in a stable way.
\end{abstract}

Keywords: quintic Powell-Sabin splines, normalized B-splines, control points, control polynomials, Bézier control net

AMS classification: 65D07, 65D17, 68U07

\section{Introduction}

Quadratic Powell-Sabin splines are defined on triangulations with a particular refinement. Each triangle in the triangulation is split into six subtriangles. These splines are $C^{1}$-continuous, and they can be represented in a normalized basis [2], in the sense that the basis functions $B_{i}, i=1, \ldots, N$, form a convex partition of unity, i.e.,

$$
B_{i}(x, y) \geq 0, \quad \text { and } \quad \sum_{i=1}^{N} B_{i}(x, y)=1,
$$

for all points $(x, y)$ in the domain. The construction of the basis allows us to define control triangles in a natural way. This normalized B-spline representation is effective in a wide range of application areas (see, e.g., $[5,8,9,13,14])$.

Several authors considered $C^{2}$-continuous quintic spline functions over triangulations of PowellSabin's type $[10,6,1,7]$. In this paper we will follow the approach by Lai and Schumaker [7], and we will construct a compact normalized basis for this spline space. Recently, a normalized basis was developed for reduced Clough-Tocher splines [12].

The paper is organized as follows. In Section 2 we recall some general concepts of polynomials on triangles, and we define the quintic Powell-Sabin splines. Section 3 describes the construction of the normalized basis, which is based on determining a set of triangles that contain a specific set of points. In Section 4 we define control points and tangent control polynomials which are useful for computer aided geometric design. We also consider a stable computation of the Bézier ordinates of the spline. Finally, we show how the values of some spline coefficients can be determined from the other spline coefficients in an appropriate way. 


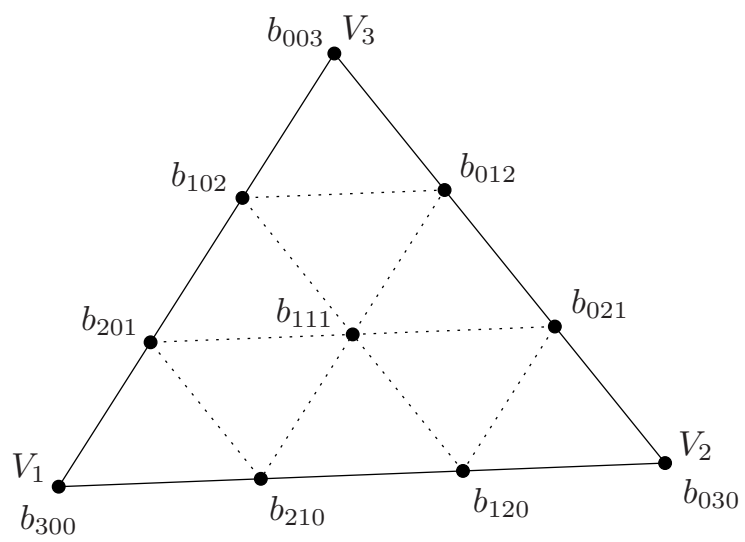

Figure 1: Schematic representation of the Bézier ordinates of a cubic bivariate polynomial.

\section{Quintic Powell-Sabin splines}

\subsection{Bivariate polynomials}

Let $\mathcal{T}\left(V_{1}, V_{2}, V_{3}\right)$ be a non-degenerated triangle. Any point $P$ in the plane of the triangle can be uniquely expressed in terms of the barycentric coordinates $\tau=\left(\tau_{1}, \tau_{2}, \tau_{3}\right)$ with respect to $\mathcal{T}$. Let $\Pi_{d}$ denote the linear space of bivariate polynomials of total degree less than or equal to $d$. Any polynomial $p_{d} \in \Pi_{d}$ on triangle $\mathcal{T}$ has a unique Bernstein-Bézier representation [4]

$$
p_{d}(\tau)=\sum_{i+j+k=d} b_{i j k} B_{i j k}^{d}(\tau)
$$

with

$$
B_{i j k}^{d}(\tau)=\frac{d !}{i ! j ! k !} \tau_{1}{ }^{i} \tau_{2}{ }^{j} \tau_{3}{ }^{k}
$$

the Bernstein polynomials of degree $d$, which form a convex partition of unity on $\mathcal{T}$. The coefficients $b_{i j k}$ are called Bézier ordinates, and the Bézier domain points $\xi_{i j k}$ are defined as the points with barycentric coordinates $\left(\frac{i}{d}, \frac{j}{d}, \frac{k}{d}\right)$. By associating each Bézier ordinate $b_{i j k}$ with the Bézier domain points $\xi_{i j k}$, we can display the Bernstein-Bézier representation schematically as in Figure 1 for the case $d=3$. The piecewise linear interpolant of the Bézier control points, defined as $\mathbf{b}_{i j k}=$ $\left(\xi_{i j k}, b_{i j k}\right)$, is called the Bézier control net. This control net is tangent to the polynomial surface at the three vertices. Polynomials in their Bernstein-Bézier representation can be evaluated in a stable way using the de Casteljau algorithm. It can also be used to derive the continuity conditions on neighbouring triangles.

The blossom (also called polar form) of a polynomial $p_{d}(\tau)$ of degree $d$ is a multi-affine symmetric polynomial $\mathcal{P}_{d}\left(\tau^{1}, \tau^{2}, \ldots, \tau^{d}\right)$ satisfying

$$
p_{d}(\tau)=\mathcal{P}_{d}(\tau, \tau, \ldots, \tau)
$$

For any polynomial there exists a unique blossom [11]. Let $\tau^{l}=\left(\tau_{1}^{l}, \tau_{2}^{l}, \tau_{3}^{l}\right), l=1,2,3$, the blossom 
of $p_{3}$ on triangle $\mathcal{T}\left(V_{1}, V_{2}, V_{3}\right)$ is given by

$$
\begin{aligned}
\mathcal{P}_{3}\left(\tau^{1}, \tau^{2}, \tau^{3}\right)= & b_{300}\left(\tau_{1}^{1} \tau_{1}^{2} \tau_{1}^{3}\right)+b_{030}\left(\tau_{2}^{1} \tau_{2}^{2} \tau_{2}^{3}\right)+b_{003}\left(\tau_{3}^{1} \tau_{3}^{2} \tau_{3}^{3}\right) \\
& +b_{210}\left(\tau_{2}^{1} \tau_{1}^{2} \tau_{1}^{3}+\tau_{1}^{1} \tau_{2}^{2} \tau_{1}^{3}+\tau_{1}^{1} \tau_{1}^{2} \tau_{2}^{3}\right)+b_{201}\left(\tau_{3}^{1} \tau_{1}^{2} \tau_{1}^{3}+\tau_{1}^{1} \tau_{3}^{2} \tau_{1}^{3}+\tau_{1}^{1} \tau_{1}^{2} \tau_{3}^{3}\right) \\
& +b_{021}\left(\tau_{3}^{1} \tau_{2}^{2} \tau_{2}^{3}+\tau_{2}^{1} \tau_{3}^{2} \tau_{2}^{3}+\tau_{2}^{1} \tau_{2}^{2} \tau_{3}^{3}\right)+b_{120}\left(\tau_{1}^{1} \tau_{2}^{2} \tau_{2}^{3}+\tau_{2}^{1} \tau_{1}^{2} \tau_{2}^{3}+\tau_{2}^{1} \tau_{2}^{2} \tau_{1}^{3}\right) \\
& +b_{102}\left(\tau_{1}^{1} \tau_{3}^{2} \tau_{3}^{3}+\tau_{3}^{1} \tau_{1}^{2} \tau_{3}^{3}+\tau_{3}^{1} \tau_{3}^{2} \tau_{1}^{3}\right)+b_{012}\left(\tau_{2}^{1} \tau_{3}^{2} \tau_{3}^{3}+\tau_{3}^{1} \tau_{2}^{2} \tau_{3}^{3}+\tau_{3}^{1} \tau_{3}^{2} \tau_{2}^{3}\right) \\
& +b_{111}\left(\tau_{1}^{1} \tau_{2}^{2} \tau_{3}^{3}+\tau_{1}^{1} \tau_{3}^{2} \tau_{2}^{3}+\tau_{2}^{1} \tau_{3}^{2} \tau_{1}^{3}+\tau_{2}^{1} \tau_{1}^{2} \tau_{3}^{3}+\tau_{3}^{1} \tau_{1}^{2} \tau_{2}^{3}+\tau_{3}^{1} \tau_{2}^{2} \tau_{1}^{3}\right),
\end{aligned}
$$

with $b_{i j k}$ the Bézier ordinates of $p_{3}$ on $\mathcal{T}$. Subdivision is very easy using blossoming. Given a second triangle, where its vertices $W_{l}, l=1,2,3$, have $\sigma^{l}=\left(\sigma_{1}^{l}, \sigma_{2}^{l}, \sigma_{3}^{l}\right)$ as barycentric coordinates with respect to the original triangle. The Bézier ordinates $d_{i j k}$ of $p_{3}$ on the new triangle are then found using (2.4) by

$$
\begin{array}{lll}
d_{300}=\mathcal{P}_{3}\left(\sigma^{1}, \sigma^{1}, \sigma^{1}\right), & d_{210}=\mathcal{P}_{3}\left(\sigma^{1}, \sigma^{1}, \sigma^{2}\right), & d_{201}=\mathcal{P}_{3}\left(\sigma^{1}, \sigma^{1}, \sigma^{3}\right), \\
d_{030}=\mathcal{P}_{3}\left(\sigma^{2}, \sigma^{2}, \sigma^{2}\right), & d_{021}=\mathcal{P}_{3}\left(\sigma^{2}, \sigma^{2}, \sigma^{3}\right), & d_{120}=\mathcal{P}_{3}\left(\sigma^{1}, \sigma^{2}, \sigma^{2}\right), \\
d_{003}=\mathcal{P}_{3}\left(\sigma^{3}, \sigma^{3}, \sigma^{3}\right), & d_{102}=\mathcal{P}_{3}\left(\sigma^{1}, \sigma^{3}, \sigma^{3}\right), & d_{012}=\mathcal{P}_{3}\left(\sigma^{2}, \sigma^{3}, \sigma^{3}\right), \\
d_{111}=\mathcal{P}_{3}\left(\sigma^{1}, \sigma^{2}, \sigma^{3}\right) . & &
\end{array}
$$

The multi-affine de Casteljau algorithm allows us to evaluate the blossom in a stable way.

\subsection{The PS5-spline space}

Consider a simply connected subset $\Omega \subset \mathbb{R}^{2}$ with polygonal boundary $\partial \Omega$. Let $\Delta$ be a conforming triangulation $\Delta$ of $\Omega$, i.e., no triangle contains a vertex different from its own three vertices. Let $n_{v}, n_{t}$ and $n_{e}$ be the number of vertices, triangles and edges in $\Delta$, respectively.

A Powell-Sabin (PS-) refinement $\Delta^{*}$ of the conforming triangulation $\Delta$ partitions each triangle $\mathcal{T}_{j} \in \Delta$ into six smaller triangles in the following way:

1. Choose an interior point $Z_{j}$ in each triangle $\mathcal{T}_{j}$, so that if two triangles $\mathcal{T}_{i}$ and $\mathcal{T}_{j}$ have a common edge, then the line joining $Z_{i}$ and $Z_{j}$ intersects the common edge at a point $R_{i j}$ between its vertices.

2. Join each point $Z_{j}$ to the vertices of $\mathcal{T}_{j}$.

3. For each edge of the triangle $\mathcal{T}_{j}$

(a) which is common to a triangle $\mathcal{T}_{i}$ : join $Z_{j}$ to the intersection point $R_{i j}$ of that edge and the line $Z_{i}-Z_{j}$.

(b) which belongs to the boundary $\partial \Omega$ : join $Z_{j}$ to an arbitrary point $R_{i j}$ on that edge;

The obtained subtriangles are denoted as $\mathcal{T}^{*} \in \Delta^{*}$. The space of piecewise quintic polynomials on $\Delta^{*}$ with global $C^{2}$-continuity is denoted as

$$
S_{5}^{2}\left(\Delta^{*}\right)=\left\{s \in C^{2}(\Omega):\left.s\right|_{\mathcal{T}^{*}} \in \Pi_{5}, \mathcal{T}^{*} \in \Delta^{*}\right\} .
$$

We consider a particular subspace of $S_{5}^{2}\left(\Delta^{*}\right)$ with additional smoothness around some vertices and edges. Let $\mathcal{V}=\left\{V_{i}\right\}_{i=1}^{n_{v}}$ be the set of vertices in $\Delta$, let $\mathcal{Z}^{*}=\left\{Z_{i}\right\}_{i=1}^{n_{t}}$ be the set of split points in $\Delta^{*}$, and let $\mathcal{E}^{*}$ be the set of all edges in $\Delta^{*}$ that connect a split point $Z_{i}$ to a point $R_{i j}$. The quintic Powell-Sabin (PS5-) spline space is defined as

$$
\hat{S}_{5}^{2}\left(\Delta^{*}\right)=\left\{s \in S_{5}^{2}\left(\Delta^{*}\right): s \in C^{3}(W), W \in\left(\mathcal{V} \cup \mathcal{Z}^{*}\right) ; s \in C^{3}(e), e \in \mathcal{E}^{*}\right\} .
$$




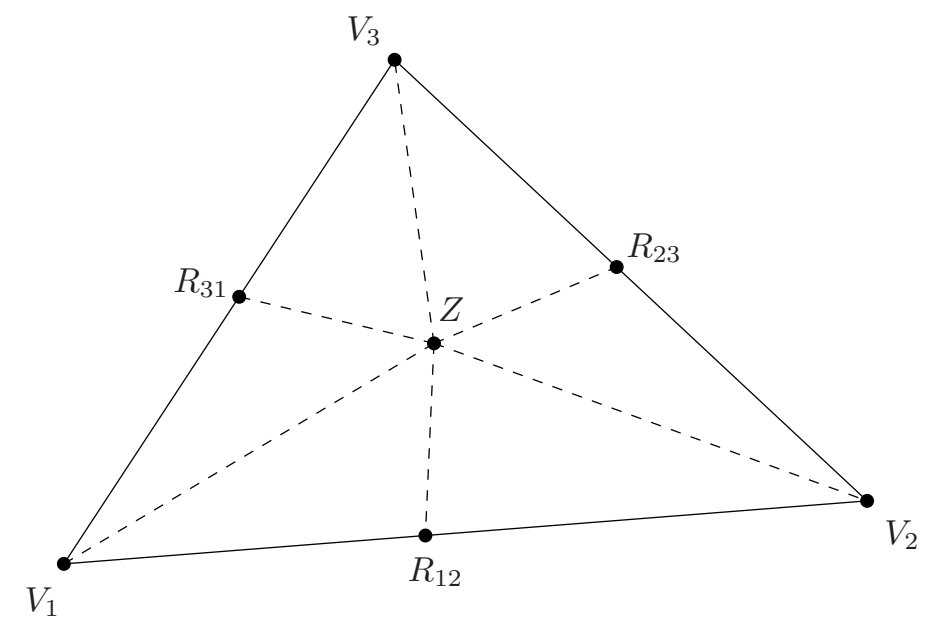

Figure 2: A Powell-Sabin split of a triangle $\mathcal{T}_{k}\left(V_{1}, V_{2}, V_{3}\right)$ drawn with dashed lines.

Here, $C^{\mu}(W)$ means that the polynomials on triangles in $\Delta^{*}$ sharing the vertex $W$ have common derivatives up to order $\mu$ at that vertex. Analogously, $C^{\mu}(e)$ means that the polynomials on triangles in $\Delta^{*}$ sharing the edge $e$ have common derivatives up to order $\mu$ along that edge. In [7] it is proved that the dimension of the space $\hat{S}_{5}^{2}\left(\Delta^{*}\right)$ equals $10 n_{v}+n_{t}$. The following interpolation problem can then be considered: there exists a unique spline $s(x, y) \in \hat{S}_{5}^{2}\left(\Delta^{*}\right)$ such that

$$
\frac{\partial^{a+b}}{\partial x^{a} \partial y^{b}} s\left(V_{l}\right)=f_{x^{a} y^{b}, l}, \quad l=1, \ldots, n_{v}, \quad a \geq 0, b \geq 0, a+b \leq 3,
$$

and

$$
s\left(Z_{m}\right)=g_{m}, \quad m=1, \ldots, n_{t},
$$

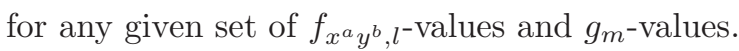

In the next section we will look for a suitable B-spline representation of $s(x, y) \in \hat{S}_{5}^{2}\left(\Delta^{*}\right)$,

$$
s(x, y)=\sum_{i=1}^{n_{v}} \sum_{j=1}^{10} c_{i, j}^{v} B_{i, j}^{v}(x, y)+\sum_{k=1}^{n_{t}} c_{k}^{t} B_{k}^{t}(x, y)
$$

in which the basis functions $B_{i, j}^{v}(x, y)$ and $B_{k}^{t}(x, y)$ have a local support and form a convex partition of unity. We shall call $B_{i, j}^{v}(x, y)$ and $B_{k}^{t}(x, y)$ a B-spline with respect to vertex $V_{i}$ and triangle $\mathcal{T}_{k}$, respectively.

\section{Normalized quintic Powell-Sabin B-splines}

\subsection{A B-spline with respect to a triangle}

We define the B-spline $B_{k}^{t}(x, y)$ with respect to triangle $\mathcal{T}_{k}$ as the unique solution of interpolation problem (2.8) with all $f_{x^{a} y^{b}, l}=0$ and with all $g_{m}=0$, except for $m=k$, where $g_{k}=\beta_{k} \neq 0$. It is easy to prove that such a spline vanishes outside $\mathcal{T}_{k}$. 


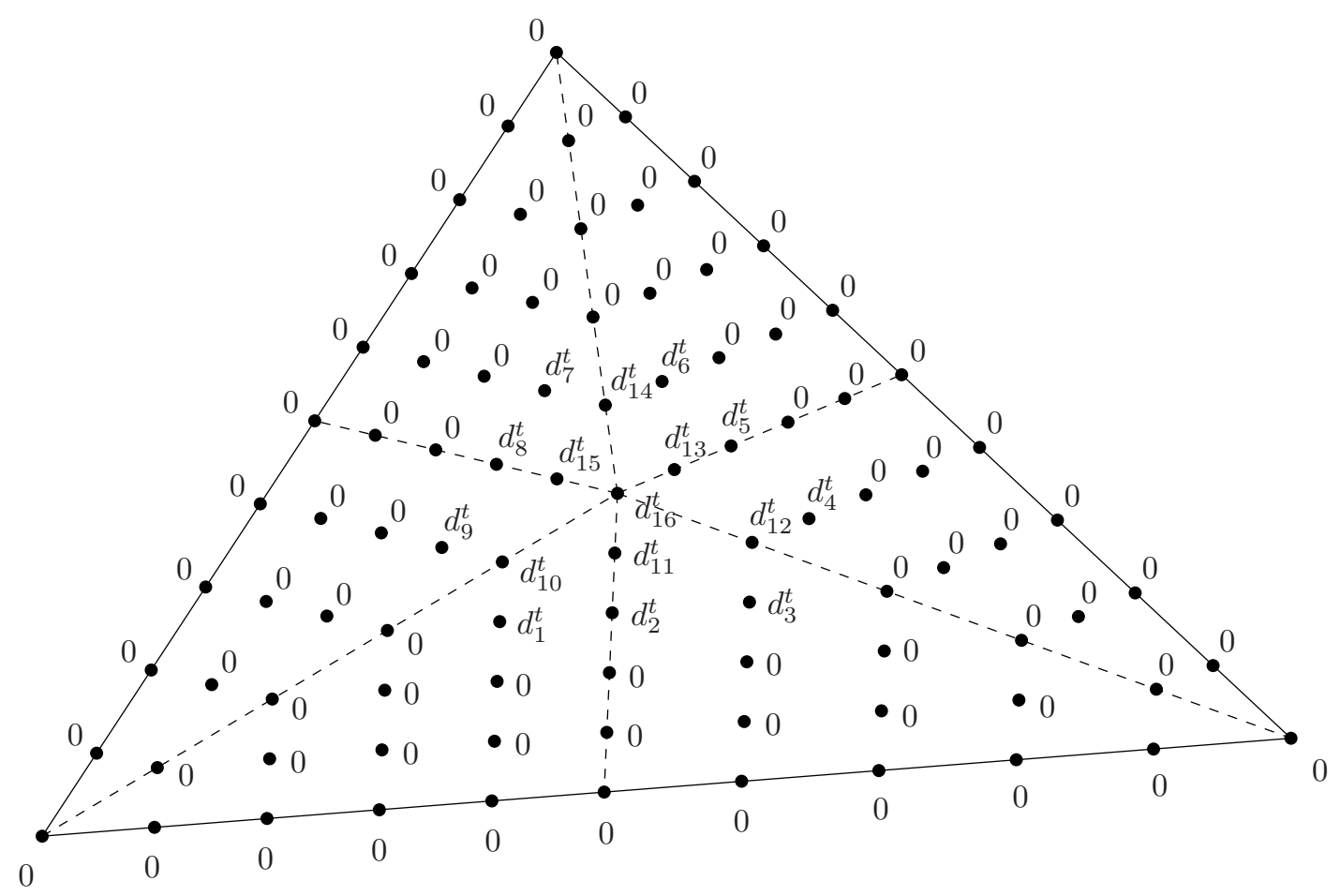

Figure 3: Schematic representation of the Bézier ordinates of a B-spline with respect to a triangle.

In order to specify the value of $\beta_{k}$, we look at the Bernstein-Bézier representation of B-spline $B_{k}^{t}(x, y)$. We consider the macro-triangle $\mathcal{T}_{k}\left(V_{1}, V_{2}, V_{3}\right)$, as shown in Figure 2 , and we assume that the points indicated in the figure have the following barycentric coordinates:

$$
\begin{aligned}
& V_{1}=(1,0,0), \quad V_{2}=(0,1,0), \quad V_{3}=(0,0,1), \quad Z=\left(z_{1}, z_{2}, z_{3}\right), \\
& R_{12}=\left(\lambda_{12}, \lambda_{21}, 0\right), \quad R_{23}=\left(0, \lambda_{23}, \lambda_{32}\right), \quad R_{31}=\left(\lambda_{13}, 0, \lambda_{31}\right) .
\end{aligned}
$$

On each micro-triangle in $\Delta^{*}$ the spline is a quintic polynomial that can be represented in its Bernstein-Bézier formulation, i.e., with $d=5$ in equations (2.1) and (2.2). The corresponding Bézier ordinates are schematically represented in Figure 3. Note that $\beta_{k}=d_{16}^{t}$. From the definition of the B-spline it follows that many of these ordinates are zero, as can be seen in the figure. The remaining Bézier ordinates are constructed in such a way that they form Bézier ordinates after subdivision of a single cubic polynomial defined on the triangle $\mathcal{T}$ with vertices

$$
P_{1}=\frac{3}{5} V_{1}+\frac{2}{5} Z, \quad P_{2}=\frac{3}{5} V_{2}+\frac{2}{5} Z, \quad P_{3}=\frac{3}{5} V_{3}+\frac{2}{5} Z
$$

This construction ensures that the B-spline is $C^{3}$-continuous at split point $Z$. We take the cubic polynomial $p_{3}(\tau)$ in Bernstein-Bézier representation (see Figure 1) with

$$
\begin{aligned}
& b_{300}=0, \quad b_{210}=0, \quad b_{201}=0, \quad b_{030}=0, \quad b_{021}=0, \quad b_{120}=0, \\
& b_{003}=0, \quad b_{102}=0, \quad b_{012}=0, \quad b_{111}=1 .
\end{aligned}
$$

We consider the PS-refinement of triangle $\mathcal{T}\left(P_{1}, P_{2}, P_{3}\right)$ where the points have the same barycentric coordinates as in (3.1). We then compute the Bézier ordinates of $p_{3}(\tau)$ on this refinement using 


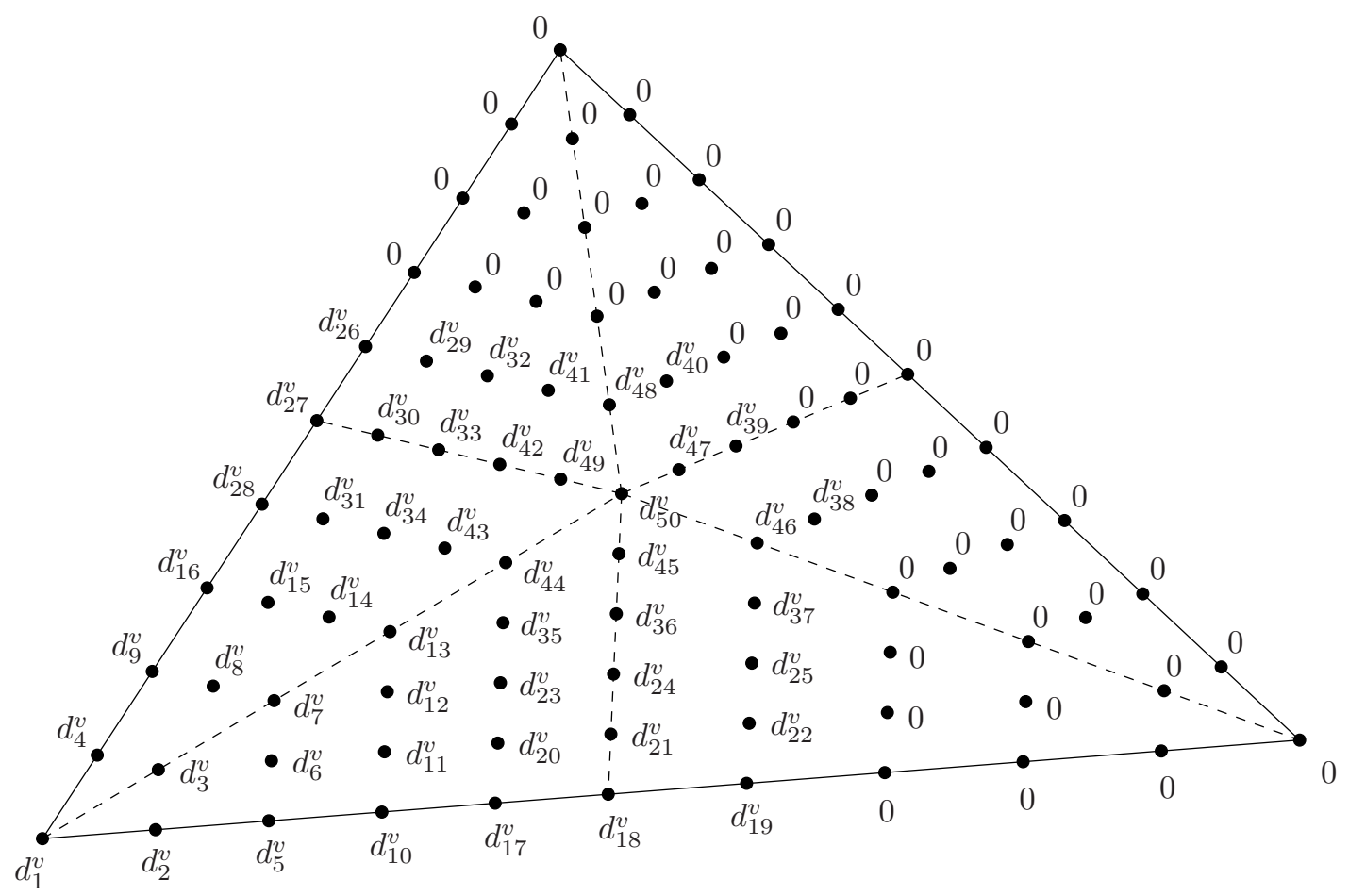

Figure 4: Schematic representation of the Bézier ordinates of a B-spline with respect to a vertex.

the formulae (2.4)-(2.5), and we obtain the values

$$
\begin{aligned}
& d_{1}^{t}=\lambda_{21} z_{3}, \quad d_{2}^{t}=2 \lambda_{12} \lambda_{21} z_{3}, \quad d_{3}^{t}=\lambda_{12} z_{3}, \\
& d_{10}^{t}=2 z_{2} z_{3}, \quad d_{11}^{t}=2 z_{3}\left(\lambda_{12} z_{2}+\lambda_{21} z_{1}\right), \quad d_{12}^{t}=2 z_{1} z_{3}, \\
& d_{16}^{t}=6 z_{1} z_{2} z_{3} .
\end{aligned}
$$

Similar expressions hold for the other Bézier ordinates of $B_{k}^{t}(x, y)$. Since all its Bézier ordinates are nonnegative, the B-spline is nonnegative.

\subsection{A B-spline with respect to a vertex}

The molecule (also called 1-ring) $M_{i}$ of vertex $V_{i}$ is defined as the union of all triangles in the triangulation that contain $V_{i}$. The B-spline $B_{i, j}^{v}(x, y)$ with respect to vertex $V_{i}$ is defined as the unique solution of interpolation problem (2.8) with all $f_{x^{a} y^{b}, l}=0$, except for $l=i$, where $f_{x^{a} y^{b}, i}=\alpha_{i, j}^{a b}$, and with all $g_{m}=0$, except for any $m$ such that $\mathcal{T}_{m} \in M_{i}$, where $g_{m}=\beta_{i, j}^{m}$. Such a spline is zero outside the molecule of vertex $V_{i}$. In order to have a unique spline representation (2.9), we must choose for each vertex ten decuples $\left\{\alpha_{i, j}^{a b}, 0 \leq a+b \leq 3\right\}, j=1, \ldots, 10$, which form a set of ten linearly independent vectors.

We consider again the macro-triangle $\mathcal{T}_{k}\left(V_{1}, V_{2}, V_{3}\right)$ depicted in Figure 2. The barycentric coordinates of the points in the figure are given in (3.1). We use the Bernstein-Bézier representation of B-spline $B_{1, j}^{v}(x, y)$ corresponding to vertex $V_{1}$, in order to specify the value of $\beta_{1, j}^{k}$ given the decuple $\left\{\alpha_{1, j}^{a b}, 0 \leq a+b \leq 3\right\}$. The corresponding Bézier ordinates are schematically represented 
in Figure 4. From the definition of the B-spline we get many ordinates that are zero. Because of the $C^{3}$-smoothness of the spline at $V_{1}$, the ordinates $d_{1}^{v}, \ldots, d_{16}^{v}$ are uniquely determined by the values of $\alpha_{1, j}^{a b}$. Later on, in $\S 4.2$, we discuss this in more detail. Using the $C^{3}$-smoothness conditions across the edge $Z-R_{12}$, we obtain the ordinates $d_{17}^{v}, \ldots, d_{25}^{v}$. Defining the values

$$
\tilde{d}_{10}^{v}=\frac{d_{10}^{v}-\lambda_{12} d_{5}^{v}}{\lambda_{21}}, \quad \tilde{d}_{11}^{v}=\frac{d_{11}^{v}-\lambda_{12} d_{6}^{v}}{\lambda_{21}}, \quad \tilde{d}_{12}^{v}=\frac{d_{12}^{v}-\lambda_{12} d_{7}^{v}}{\lambda_{21}},
$$

we get

$$
\begin{array}{lll}
d_{17}^{v}=\lambda_{12}\left(d_{10}^{v}+\lambda_{21} \tilde{d}_{10}^{v}\right), & d_{18}^{v}=\lambda_{12}^{2}\left(d_{10}^{v}+2 \lambda_{21} \tilde{d}_{10}^{v}\right), & d_{19}^{v}=\lambda_{12}{ }^{2} \tilde{d}_{10}^{v}, \\
d_{20}^{v}=\lambda_{12}\left(d_{11}^{v}+\lambda_{21} \tilde{d}_{11}^{v}\right), & d_{21}^{v}=\lambda_{12}{ }^{2}\left(d_{11}^{v}+2 \lambda_{21} \tilde{d}_{11}^{v}\right), & d_{22}^{v}=\lambda_{12}{ }^{2} \tilde{d}_{11}^{v} \\
d_{23}^{v}=\lambda_{12}\left(d_{12}^{v}+\lambda_{21} \tilde{d}_{12}^{v}\right), & d_{24}^{v}=\lambda_{12}{ }^{2}\left(d_{12}^{v}+2 \lambda_{21} \tilde{d}_{12}^{v}\right), & d_{25}^{v}=\lambda_{12}{ }^{2} \tilde{d}_{12}^{v}
\end{array}
$$

In a similar way we can compute the ordinates $d_{26}^{v}, \ldots, d_{34}^{v}$, using

$$
\tilde{d}_{14}^{v}=\frac{d_{14}^{v}-\lambda_{13} d_{7}^{v}}{\lambda_{31}}, \quad \tilde{d}_{15}^{v}=\frac{d_{15}^{v}-\lambda_{13} d_{8}^{v}}{\lambda_{31}}, \quad \tilde{d}_{16}^{v}=\frac{d_{16}^{v}-\lambda_{13} d_{9}^{v}}{\lambda_{31}} .
$$

The remaining Bézier ordinates must be chosen such that the B-spline is $C^{3}$-continuous at split point $Z$. Therefore, we take the values of $d_{7}^{v}, d_{12}^{v}, d_{13}^{v}, d_{14}^{v}, d_{23}^{v}, d_{24}^{v}, d_{25}^{v}, d_{32}^{v}, \ldots, d_{50}^{v}$ as the Bézier ordinates after subdivision of a single cubic polynomial defined on the triangle with vertices (3.2). We consider the cubic polynomial $p_{3}(\tau)$ in Bernstein-Bézier representation (see Figure 1) with

$$
\begin{aligned}
& b_{300}=d_{7}^{v}, \quad b_{210}=\tilde{d}_{12}^{v}, \quad b_{201}=\tilde{d}_{14}^{v}, \quad b_{030}=0, \quad b_{021}=0, \quad b_{120}=0, \\
& b_{003}=0, \quad b_{102}=0, \quad b_{012}=0, \quad b_{111}=0 .
\end{aligned}
$$

Following the same procedure as in $\S 3.1$, we compute the Bézier ordinates of $p_{3}(\tau)$ on the particular PS-refinement where the points have barycentric coordinates identical to (3.1). Using the formulae (2.4)-(2.5), we get

$$
\begin{aligned}
& d_{35}^{v}=\lambda_{12} d_{13}^{v}+\lambda_{21} z_{1} \tilde{d}_{12}^{v}, \quad d_{36}^{v}=\lambda_{12}\left(\lambda_{12} d_{13}^{v}+2 \lambda_{21} z_{1} \tilde{d}_{12}^{v}\right), \quad d_{37}^{v}=\lambda_{12} z_{1} \tilde{d}_{12}^{v}, \\
& d_{38}^{v}=0, \quad d_{39}^{v}=0, \quad d_{40}^{v}=0, \\
& d_{44}^{v}=z_{1}\left(d_{13}^{v}+z_{2} \tilde{d}_{12}^{v}+z_{3} \tilde{d}_{14}^{v}\right), \quad d_{45}^{v}=z_{1}\left(\lambda_{12}\left(d_{13}^{v}+z_{2} \tilde{d}_{12}^{v}+z_{3} \tilde{d}_{14}^{v}\right)+\lambda_{21} z_{1} \tilde{d}_{12}^{v}\right), \\
& d_{46}^{v}=z_{1}^{2} \tilde{d}_{12}^{v}, \quad d_{47}^{v}=z_{1}^{2}\left(\lambda_{23} \tilde{d}_{12}^{v}+\lambda_{32} \tilde{d}_{14}^{v}\right), \\
& d_{50}^{v}=z_{1}^{2}\left(d_{13}^{v}+2 z_{2} \tilde{d}_{12}^{v}+2 z_{3} \tilde{d}_{14}^{v}\right) .
\end{aligned}
$$

We find expressions similar to (3.7) for the Bézier ordinates $d_{41}^{v}, d_{42}^{v}, d_{43}^{v}, d_{48}^{v}$ and $d_{49}^{v}$. It holds that $\beta_{1, j}^{k}=d_{50}^{v}$.

To obtain conditions ensuring nonnegativity, it is sufficient to impose that all Bézier ordinates of the B-spline $B_{1, j}^{v}(x, y)$ on triangle $\mathcal{T}_{k}\left(V_{1}, V_{2}, V_{3}\right)$ are nonnegative. Looking at the formulae (3.4)-(3.7), this is the case when

$$
d_{l}^{v} \geq 0, \quad l=1, \ldots, 9,
$$

and

$$
\tilde{d}_{10}^{v} \geq 0, \quad \tilde{d}_{11}^{v} \geq 0, \quad \tilde{d}_{12}^{v} \geq 0, \quad \tilde{d}_{14}^{v} \geq 0, \quad \tilde{d}_{15}^{v} \geq 0, \quad \tilde{d}_{16}^{v} \geq 0 .
$$

If the molecule of vertex $V_{1}$ has more than one triangle, we have to impose similar conditions for each of these triangles. 


\subsection{A geometric approach to form a convex partition of unity}

We now determine for each vertex $V_{i}$ a particular set of ten linearly independent decuples $\left\{\alpha_{i, j}^{a b}, 0 \leq a+b \leq 3\right\}, j=1, \ldots, 10$, such that the corresponding B-splines $B_{i, j}^{v}(x, y)$ and $B_{k}^{t}(x, y)$ will form a convex partition of unity.

With each vertex $V_{i}$ we associate a triangle $t_{i}\left(Q_{i, 1}^{v}, Q_{i, 2}^{v}, Q_{i, 3}^{v}\right)$. Each decuple $\left\{\alpha_{i, j}^{a b}, 0 \leq a+b \leq 3\right\}$ is then related to the function and derivative values at the point $V_{i}$ of one of the Bernstein polynomials (2.2) of degree three with respect to triangle $t_{i}$. They are defined as

$$
\begin{aligned}
\alpha_{i, 1}^{a b} & =\frac{20}{(5-a-b)(4-a-b)}\left(\frac{3}{5}\right)^{a+b} \frac{\partial^{a+b}}{\partial x^{a} \partial y^{b}} B_{300}^{3}\left(V_{i}\right), \\
\alpha_{i, 2}^{a b} & =\frac{20}{(5-a-b)(4-a-b)}\left(\frac{3}{5}\right)^{a+b} \frac{\partial^{a+b}}{\partial x^{a} \partial y^{b}} B_{030}^{3}\left(V_{i}\right), \\
\alpha_{i, 3}^{a b} & =\frac{20}{(5-a-b)(4-a-b)}\left(\frac{3}{5}\right)^{a+b} \frac{\partial^{a+b}}{\partial x^{a} \partial y^{b}} B_{003}^{3}\left(V_{i}\right), \\
\alpha_{i, 4}^{a b} & =\frac{20}{(5-a-b)(4-a-b)}\left(\frac{3}{5}\right)^{a+b} \frac{\partial^{a+b}}{\partial x^{a} \partial y^{b}} B_{210}^{3}\left(V_{i}\right), \\
& \ldots, \quad\left(\frac{3}{5}\right)^{a+b} \frac{\partial^{a+b}}{\partial x^{a} \partial y^{b}} B_{111}^{3}\left(V_{i}\right),
\end{aligned}
$$

for all $0 \leq a+b \leq 3$. The triangle $t_{i}$ shall be called the PS5-triangle with respect to $V_{i}$. The blossom of the Bernstein polynomial corresponding to decuple $\left\{\alpha_{i, j}^{a b}, 0 \leq a+b \leq 3\right\}$ in (3.9) is denoted by $\mathcal{B}_{i, j}\left(\tau^{1}, \tau^{2}, \tau^{3}\right)$.

Property 3.1. The B-splines $B_{k}^{t}(x, y)$ and $B_{i, j}^{v}(x, y)$ constructed using a set of PS5-triangles form a partition of unity.

Proof. From the definition of the B-splines it follows that only ten basis functions have function and derivative values at vertex $V_{i}$ that are not all zero. We also know that the Bernstein polynomials in (3.9) form a partition of unity on $t_{i}$. We then immediately obtain that

$$
\sum_{j=1}^{10} \alpha_{i, j}^{00}=1
$$

and

$$
\sum_{j=1}^{10} \alpha_{i, j}^{a b}=0, \quad 1 \leq a+b \leq 3
$$

for $i=1, \ldots, n_{v}$. We now show that

$$
\beta_{k}+\sum_{i \mid \mathcal{T}_{k} \in M_{i}} \sum_{j=1}^{10} \beta_{i, j}^{k}=1
$$

for $k=1, \ldots, n_{t}$. We consider the triangle $\mathcal{T}_{k}\left(V_{1}, V_{2}, V_{3}\right)$ depicted in Figure 2. There are 31 
B-splines that have a nonzero function value at split point $Z$. Using (3.3), (3.7) and (3.10), we get

$$
\begin{aligned}
& \beta_{k}=6 z_{1} z_{2} z_{3}, \\
& \sum_{j=1}^{10} \beta_{1, j}^{k}=z_{1}^{2}\left(1+2 z_{2}+2 z_{3}\right), \\
& \sum_{j=1}^{10} \beta_{2, j}^{k}=z_{2}^{2}\left(1+2 z_{3}+2 z_{1}\right), \\
& \sum_{j=1}^{10} \beta_{3, j}^{k}=z_{3}^{2}\left(1+2 z_{1}+2 z_{2}\right) .
\end{aligned}
$$

They sum up to one, since $z_{1}+z_{2}+z_{3}=1$ and

$$
\begin{aligned}
& 6 z_{1} z_{2} z_{3}+z_{1}^{2}\left(1+2 z_{2}+2 z_{3}\right)+z_{2}^{2}\left(1+2 z_{3}+2 z_{1}\right)+z_{3}^{2}\left(1+2 z_{1}+2 z_{2}\right) \\
& \quad=z_{1}^{2}+z_{2}^{2}+z_{3}^{2}+2\left(z_{1} z_{2}+z_{2} z_{3}+z_{3} z_{1}\right)\left(z_{1}+z_{2}+z_{3}\right) \\
& \quad=\left(z_{1}+z_{2}+z_{3}\right)^{2}=1
\end{aligned}
$$

The proof is completed by considering interpolation problem (2.8) and (3.10)-(3.11).

We now give a geometric approach to satisfy the nonnegativity conditions (3.8). We will request that a specific set of points must be inside each PS5-triangle. These points shall be called PS5points.

Property 3.2. The B-splines $B_{k}^{t}(x, y)$ and $B_{i, j}^{v}(x, y)$ constructed using a set of PS5-triangles $t_{i}$ are nonnegative, when each $t_{i}$ contains its corresponding vertex $V_{i}$ and the points

$$
S_{i l}=\frac{2}{5} V_{i}+\frac{3}{5} V_{l}
$$

for all vertices $V_{l}$ that are situated at the boundary of the molecule $M_{i}$ of $V_{i}$.

Proof. By (3.3) we see that all Bézier ordinates of B-spline $B_{k}^{t}(x, y)$ are nonnegative. Hence, the $\mathrm{B}$-spline with respect to triangle $\mathcal{T}_{k}$ is nonnegative. The remaining part of the proof is devoted to the B-splines with respect to a vertex.

We first show that relation (3.9) implies that the Bézier ordinates of B-spline $B_{i, j}^{v}(x, y)$ in the neighbourhood of vertex $V_{i}$ can be regarded as Bézier ordinates of a certain Bernstein polynomial of degree three with respect to PS5-triangle $t_{i}$ after applying subdivision. We consider again the triangle $\mathcal{T}_{k}\left(V_{1}, V_{2}, V_{3}\right)$ shown in Figure 2, and the schematic Bernstein-Bézier representation of $B_{1, j}^{v}(x, y)$ in Figure 4 . Let $u_{1}$ and $u_{2}$ be the unit vectors defined by

$$
u_{1}=\frac{R_{12}-V_{1}}{\left\|R_{12}-V_{1}\right\|}, \quad u_{2}=\frac{Z-V_{1}}{\left\|Z-V_{1}\right\|} .
$$

A cubic polynomial $p_{3}$ defined on the triangle with vertices

$$
P_{1}=V_{1}, \quad P_{2}=\frac{2}{5} V_{1}+\frac{3}{5} R_{12}, \quad P_{3}=\frac{2}{5} V_{1}+\frac{3}{5} Z,
$$


has the values $d_{1}^{v}, d_{2}^{v}, d_{3}^{v}, d_{5}^{v}, d_{6}^{v}, d_{7}^{v}, d_{10}^{v}, d_{11}^{v}, d_{12}^{v}$ and $d_{13}^{v}$ as Bézier ordinates, if and only if

$$
\begin{aligned}
& p_{3}\left(V_{1}\right)=B_{1, j}^{v}\left(V_{1}\right)=d_{1}^{v}, \\
& \frac{1}{3} \frac{3}{5} \frac{\partial}{\partial u_{1}} p_{3}\left(V_{1}\right)=\frac{1}{5} \frac{\partial}{\partial u_{1}} B_{1, j}^{v}\left(V_{1}\right)=\frac{d_{2}^{v}-d_{1}^{v}}{\left\|R_{12}-V_{1}\right\|}, \\
& \frac{1}{3} \frac{3}{5} \frac{\partial}{\partial u_{2}} p_{3}\left(V_{1}\right)=\frac{1}{5} \frac{\partial}{\partial u_{2}} B_{1, j}^{v}\left(V_{1}\right)=\frac{d_{3}^{v}-d_{1}^{v}}{\left\|Z-V_{1}\right\|}, \\
& \frac{1}{6}\left(\frac{3}{5}\right)^{2} \frac{\partial^{2}}{\partial u_{1}^{2}} p_{3}\left(V_{1}\right)=\frac{1}{20} \frac{\partial^{2}}{\partial u_{1}^{2}} B_{1, j}^{v}\left(V_{1}\right)=\frac{d_{5}^{v}-2 d_{2}^{v}+d_{1}^{v}}{\left\|R_{12}-V_{1}\right\|^{2}}, \\
& \frac{1}{6}\left(\frac{3}{5}\right)^{2} \frac{\partial^{2}}{\partial u_{1} \partial u_{2}} p_{3}\left(V_{1}\right)=\frac{1}{20} \frac{\partial^{2}}{\partial u_{1} \partial u_{2}} B_{1, j}^{v}\left(V_{1}\right)=\frac{d_{6}^{v}-d_{3}^{v}-d_{2}^{v}+d_{1}^{v}}{\left\|R_{12}-V_{1}\right\|\left\|Z-V_{1}\right\|}, \\
& \frac{1}{6}\left(\frac{3}{5}\right)^{2} \frac{\partial^{2}}{\partial u_{2}^{2}} p_{3}\left(V_{1}\right)=\frac{1}{20} \frac{\partial^{2}}{\partial u_{2}{ }^{2}} B_{1, j}^{v}\left(V_{1}\right)=\frac{d_{7}^{v}-2 d_{3}^{v}+d_{1}^{v}}{\left\|Z-V_{1}\right\|^{2}}, \\
& \frac{1}{6}\left(\frac{3}{5}\right)^{3} \frac{\partial^{3}}{\partial u_{1}{ }^{3}} p_{3}\left(V_{1}\right)=\frac{1}{60} \frac{\partial^{3}}{\partial u_{1}{ }^{3}} B_{1, j}^{v}\left(V_{1}\right)=\frac{d_{10}^{v}-3 d_{5}^{v}+3 d_{2}^{v}-d_{1}^{v}}{\left\|R_{12}-V_{1}\right\|^{3}}, \\
& \frac{1}{6}\left(\frac{3}{5}\right)^{3} \frac{\partial^{3}}{\partial u_{1}^{2} \partial u_{2}} p_{3}\left(V_{1}\right)=\frac{1}{60} \frac{\partial^{3}}{\partial u_{1}^{2} \partial u_{2}} B_{1, j}^{v}\left(V_{1}\right)=\frac{d_{11}^{v}-2 d_{6}^{v}-d_{5}^{v}+d_{3}^{v}+2 d_{2}^{v}-d_{1}^{v}}{\left\|R_{12}-V_{1}\right\|^{2}\left\|Z-V_{1}\right\|}, \\
& \frac{1}{6}\left(\frac{3}{5}\right)^{3} \frac{\partial^{3}}{\partial u_{1} \partial u_{2}{ }^{2}} p_{3}\left(V_{1}\right)=\frac{1}{60} \frac{\partial^{3}}{\partial u_{1} \partial u_{2}{ }^{2}} B_{1, j}^{v}\left(V_{1}\right)=\frac{d_{12}^{v}-d_{7}^{v}-2 d_{6}^{v}+2 d_{3}^{v}+d_{2}^{v}-d_{1}^{v}}{\left\|R_{12}-V_{1}\right\|\left\|Z-V_{1}\right\|^{2}}, \\
& \frac{1}{6}\left(\frac{3}{5}\right)^{3} \frac{\partial^{3}}{\partial u_{2}{ }^{3}} p_{3}\left(V_{1}\right)=\frac{1}{60} \frac{\partial^{3}}{\partial u_{2}{ }^{3}} B_{1, j}^{v}\left(V_{1}\right)=\frac{d_{13}^{v}-3 d_{7}^{v}+3 d_{3}^{v}-d_{1}^{v}}{\left\|Z-V_{1}\right\|^{3}} .
\end{aligned}
$$

From (3.9) it follows that polynomial $p_{3}$ must be equal to a certain Bernstein polynomial of degree three with respect to PS5-triangle $t_{i}$.

Let $\sigma^{l}=\left(\sigma_{1}^{l}, \sigma_{2}^{l}, \sigma_{3}^{l}\right), l=1,2,3$, be the barycentric coordinates of the points $P_{l}$ in (3.13) with respect to PS5-triangle $t_{1}$. Note that these points can be written as

$$
P_{1}=V_{1}, \quad P_{2}=\lambda_{12} V_{1}+\lambda_{21} S_{12}, \quad P_{3}=z_{1} V_{1}+z_{2} S_{12}+z_{3} S_{13} .
$$

Referring to (2.5), we can compute the Bézier ordinates of B-spline $B_{1, j}^{v}(x, y)$ in the neighbourhood of vertex $V_{1}$ using the blossom $\mathcal{B}_{1, j}\left(\tau^{1}, \tau^{2}, \tau^{3}\right)$ of the corresponding Bernstein polynomial with respect to $t_{i}$, e.g.,

$$
\begin{array}{ll}
d_{1}^{v}=\mathcal{B}_{1, j}\left(\sigma^{1}, \sigma^{1}, \sigma^{1}\right), & d_{2}^{v}=\mathcal{B}_{1, j}\left(\sigma^{1}, \sigma^{1}, \sigma^{2}\right), \\
d_{5}^{v}=\mathcal{B}_{1, j}\left(\sigma^{1}, \sigma^{2}, \sigma^{2}\right), & d_{10}^{v}=\mathcal{B}_{1, j}\left(\sigma^{2}, \sigma^{2}, \sigma^{2}\right) .
\end{array}
$$

By the multi-affinity of the blossom we obtain that $\tilde{d}_{10}^{v}$ in (3.4) can be computed as

$$
\tilde{d}_{10}^{v}=\frac{\mathcal{B}_{1, j}\left(\sigma^{2}, \sigma^{2}, \sigma^{2}\right)-\lambda_{12} \mathcal{B}_{1, j}\left(\sigma^{1}, \sigma^{2}, \sigma^{2}\right)}{\lambda_{21}}=\mathcal{B}_{1, j}\left(\frac{\sigma^{2}-\lambda_{12} \sigma^{1}}{\lambda_{21}}, \sigma^{2}, \sigma^{2}\right) .
$$

Analogously, we have

$$
\tilde{d}_{11}^{v}=\mathcal{B}_{1, j}\left(\frac{\sigma^{2}-\lambda_{12} \sigma^{1}}{\lambda_{21}}, \sigma^{2}, \sigma^{3}\right), \quad \tilde{d}_{12}^{v}=\mathcal{B}_{1, j}\left(\frac{\sigma^{2}-\lambda_{12} \sigma^{1}}{\lambda_{21}}, \sigma^{3}, \sigma^{3}\right) .
$$

The barycentric coordinates $\left(\sigma^{2}-\lambda_{12} \sigma^{1}\right) / \lambda_{21}$ correspond to the point $S_{12}$, since

$$
S_{12}=\frac{2}{5} V_{1}+\frac{3}{5} V_{2}=\frac{P_{2}-\lambda_{12} P_{1}}{\lambda_{21}}
$$


We get expressions similar to (3.16)-(3.17) for $\tilde{d}_{14}^{v}, \tilde{d}_{15}^{v}$ and $\tilde{d}_{16}^{v}$.

Each Bernstein polynomial is nonnegative on its domain triangle. It follows that the blossom $\mathcal{B}_{1, j}\left(\tau^{1}, \tau^{2}, \tau^{3}\right)$ is nonnegative whenever the barycentric coordinates $\tau^{1}, \tau^{2}$ and $\tau^{3}$ are nonnegative. Such barycentric coordinates correspond to points that are situated inside PS5-triangle $t_{1}$. Using (3.15)-(3.17), the nonnegativity conditions (3.8) for B-spline $B_{1, j}^{v}(x, y)$ on triangle $\mathcal{T}_{k}\left(V_{1}, V_{2}, V_{3}\right)$ are satisfied when PS5-triangle $t_{1}$ contains the points $V_{1}, S_{12}$ and $S_{13}$. A similar argument can be used to ensure nonnegativity on the other triangles in the molecule of vertex $V_{1}$.

The construction of a normalized PS5-basis is reduced to finding a set of triangles that must contain a number of specified points. It is clear that this geometric problem always has a solution. There are an infinite number of solutions. An appropriate choice, as suggested in [2] for PS-triangles, is to calculate triangles of minimal area, the so-called optimal triangles.

\subsection{Optimal PS5-triangles}

We now look in more detail how we can compute optimal PS5-triangles. Computationally, such a problem leads to a quadratic programming problem. We first consider the following relation between the triplets $\left(\gamma_{i, j}, \gamma_{i, j}^{x}, \gamma_{i, j}^{y}\right), j=1,2,3$, the PS5-triangle points $Q_{i, j}^{v}=\left(Y_{i, j}^{v}, Y_{i, j}^{v}\right), j=1,2,3$, and vertex $V_{i}=\left(x_{i}, y_{i}\right)$ :

$$
\left[\begin{array}{ccc}
\gamma_{i, 1} & \gamma_{i, 2} & \gamma_{i, 3} \\
\gamma_{i, 1}^{x} & \gamma_{i, 2}^{x} & \gamma_{i, 3}^{x} \\
\gamma_{i, 1}^{y} & \gamma_{i, 2}^{y} & \gamma_{i, 3}^{y}
\end{array}\right]\left[\begin{array}{ccc}
X_{i, 1}^{v} & Y_{i, 1}^{v} & 1 \\
X_{i, 2}^{v} & Y_{i, 2}^{v} & 1 \\
X_{i, 3}^{v} & Y_{i, 3}^{v} & 1
\end{array}\right]=\left[\begin{array}{ccc}
x_{i} & y_{i} & 1 \\
1 & 0 & 0 \\
0 & 1 & 0
\end{array}\right] .
$$

Property 3.3. The construction of an optimal PS5-triangle $t_{i}$ with respect to vertex $V_{i}=\left(x_{i}, y_{i}\right)$ is equivalent to the quadratic programming problem

$$
\max \left(\gamma_{i, 1}^{x} \gamma_{i, 2}^{y}-\gamma_{i, 1}^{y} \gamma_{i, 2}^{x}\right),
$$

subjected to the linear constraints

$$
\begin{aligned}
& \gamma_{i, 1}+\gamma_{i, 2}+\gamma_{i, 3}=1, \\
& \gamma_{i, 1}^{x}+\gamma_{i, 2}^{x}+\gamma_{i, 3}^{x}=0, \\
& \gamma_{i, 1}^{y}+\gamma_{i, 2}^{y}+\gamma_{i, 3}^{y}=0,
\end{aligned}
$$

and

$$
\begin{aligned}
& \gamma_{i, j} \geq 0, \\
& L_{i l, j}:=\gamma_{i, j}+\frac{3}{5}\left(\gamma_{i, j}^{x}\left(x_{l}-x_{i}\right)+\gamma_{i, j}^{y}\left(y_{l}-y_{i}\right)\right) \geq 0,
\end{aligned}
$$

with $j=1,2,3$, and for all vertices $V_{l}=\left(x_{l}, y_{l}\right)$ that are situated at the boundary of molecule $M_{i}$ of $V_{i}$. Here, $\left(\gamma_{i, 1}, \gamma_{i, 2}, \gamma_{i, 3}\right)$ and $\left(L_{i l, 1}, L_{i l, 2}, L_{i l, 3}\right)$ are the barycentric coordinates with respect to PS5-triangle $t_{i}$ of the PS5-points $V_{i}$ and $S_{i l}$, respectively.

Proof. We first look at the objective function (3.19). Using (3.18) we obtain after some elementary calculations that

$$
\left|\begin{array}{ccc}
X_{i, 1} & Y_{i, 1} & 1 \\
X_{i, 2} & Y_{i, 2} & 1 \\
X_{i, 3} & Y_{i, 3} & 1
\end{array}\right|=\left|\begin{array}{ccc}
\gamma_{i, 1} & \gamma_{i, 2} & \gamma_{i, 3} \\
\gamma_{i, 1}^{x} & \gamma_{i, 2}^{x} & \gamma_{i, 3}^{x} \\
\gamma_{i, 1}^{y} & \gamma_{i, 2}^{y} & \gamma_{i, 3}^{y}
\end{array}\right|^{-1}=\frac{1}{\gamma_{i, 1}^{x} \gamma_{i, 2}^{y}-\gamma_{i, 1}^{y} \gamma_{i, 2}^{x}}
$$


Minimizing the area of PS5-triangle $t_{i}$ is equivalent to maximizing $\left|\gamma_{i, 1}^{x} \gamma_{i, 2}^{y}-\gamma_{i, 1}^{y} \gamma_{i, 2}^{x}\right|$. This can be relaxed to (3.19). Suppose that the triplets $\left(\gamma_{i, j}^{*}, \gamma_{i, j}^{x *}, \gamma_{i, j}^{y *}\right), j=1,2,3$, satisfy (3.20)-(3.21) and that $\left|\gamma_{i, 1}^{x *} \gamma_{i, 2}^{y *}-\gamma_{i, 1}^{y *} \gamma_{i, 2}^{x *}\right|$ is maximal with $\gamma_{i, 1}^{x *} \gamma_{i, 2}^{y *}-\gamma_{i, 1}^{y *} \gamma_{i, 2}^{x *}<0$. Then, there exists another set of triplets $\left(\hat{\gamma}_{i, j}^{*}, \hat{\gamma}_{i, j}^{x *}, \hat{\gamma}_{i, j}^{y *}\right), j=1,2,3$, with $\hat{\gamma}_{i, 1}^{*}=\gamma_{i, 2}^{*}, \hat{\gamma}_{i, 2}^{*}=\gamma_{i, 1}^{*}, \hat{\gamma}_{i, 3}^{*}=\gamma_{i, 3}^{*}, \hat{\gamma}_{i, 1}^{x *}=\gamma_{i, 2}^{x *}, \hat{\gamma}_{i, 2}^{x *}=\gamma_{i, 1}^{x *}$, $\hat{\gamma}_{i, 3}^{x *}=\gamma_{i, 3}^{x *}, \hat{\gamma}_{i, 1}^{y *}=\gamma_{i, 2}^{y *}, \hat{\gamma}_{i, 2}^{y *}=\gamma_{i, 1}^{y *}$ and $\hat{\gamma}_{i, 3}^{y *}=\gamma_{i, 3}^{y *}$. These triplets also satisfy (3.20)-(3.21) and it holds that $\hat{\gamma}_{i, 1}^{x *} \hat{\gamma}_{i, 2}^{y *}-\hat{\gamma}_{i, 1}^{y *} \hat{\gamma}_{i, 2}^{x *}>0$ and $\left|\hat{\gamma}_{i, 1}^{x *} \hat{\gamma}_{i, 2}^{y *}-\hat{\gamma}_{i, 1}^{y *} \hat{\gamma}_{i, 2}^{x *}\right|=\left|\gamma_{i, 1}^{x *} \gamma_{i, 2}^{y *}-\gamma_{i, 1}^{y *} \gamma_{i, 2}^{x *}\right|$.

We now explain the constraints (3.20)-(3.21). Let $\left(\sigma_{1}, \sigma_{2}, \sigma_{3}\right)$ be the barycentric coordinates of a point with respect to PS5-triangle $t_{1}$. Let $\left(\tau_{1}, \tau_{2}, \tau_{3}\right)$ be the barycentric coordinates of the same point with respect to triangle $\mathcal{T}\left(V_{1}, V_{2}, V_{3}\right)$ in $\Delta$. It is proved in [12] that by relation (3.18) it holds

$$
\left[\begin{array}{l}
\sigma_{1} \\
\sigma_{2} \\
\sigma_{3}
\end{array}\right]=A\left[\begin{array}{l}
\tau_{1} \\
\tau_{2} \\
\tau_{3}
\end{array}\right]
$$

with

$$
A=\left[\begin{array}{ccc}
\gamma_{1,1} & \gamma_{1,1}+\gamma_{1,1}^{x}\left(x_{2}-x_{1}\right)+\gamma_{1,1}^{y}\left(y_{2}-y_{1}\right) & \gamma_{1,1}+\gamma_{1,1}^{x}\left(x_{3}-x_{1}\right)+\gamma_{1,1}^{y}\left(y_{3}-y_{1}\right) \\
\gamma_{1,2} & \gamma_{1,2}+\gamma_{1,2}^{x}\left(x_{2}-x_{1}\right)+\gamma_{1,2}^{y}\left(y_{2}-y_{1}\right) & \gamma_{1,2}+\gamma_{1,2}^{x}\left(x_{3}-x_{1}\right)+\gamma_{1,2}^{y}\left(y_{3}-y_{1}\right) \\
\gamma_{1,3} & \gamma_{1,3}+\gamma_{1,3}^{x}\left(x_{2}-x_{1}\right)+\gamma_{1,3}^{y}\left(y_{2}-y_{1}\right) & \gamma_{1,3}+\gamma_{1,3}^{x}\left(x_{3}-x_{1}\right)+\gamma_{1,3}^{y}\left(y_{3}-y_{1}\right)
\end{array}\right] .
$$

The barycentric coordinates $\left(\tau_{1}, \tau_{2}, \tau_{3}\right)$ of the points $V_{1}, S_{12}$ and $S_{13}$ with respect to $\mathcal{T}\left(V_{1}, V_{2}, V_{3}\right)$ are $(1,0,0),(2 / 5,3 / 5,0)$ and $(2 / 5,0,3 / 5)$, respectively. Substituting these values into (3.23), we find that the barycentric coordinates $\left(\sigma_{1}, \sigma_{2}, \sigma_{3}\right)$ of these points with respect to $t_{i}$ are nothing else than the left hand sides of the inequalities (3.21). Imposing them to be nonnegative is equivalent to the condition that the points $V_{1}, S_{12}$ and $S_{13}$ are inside the PS5-triangle $t_{1}$. A similar reasoning hold for all PS5-triangles $t_{i}$ and any triangle $\mathcal{T}$ in the molecule of $V_{i}$.

In Figure 5 we illustrate the PS5-points and a few optimal PS5-triangles for a triangulation taken from $[3,12]$. Because of overlapping we have not depicted all PS5-triangles .

\section{Applications}

\subsection{Control points and control polynomials of a PS5-spline}

Since each B-spline $B_{i, j}^{v}(x, y), j=1, \ldots, 10$, with respect to vertex $V_{i}$ is related to a Bernstein polynomial of degree three, see (3.9), the corresponding coefficients $c_{i, j}^{v}$ of the PS5-spline in (2.9) can be represented schematically as in Figure 6 with respect to PS5-triangle $t_{i}$. The cubic polynomial defined on $t_{i}$ with the coefficients $c_{i, j}^{v}$ as Bézier ordinates shall be called the control polynomial with respect to vertex $V_{i}$. It shall be denoted by $T_{i}(x, y)$.

Property 4.1. The control polynomial $T_{i}(x, y)$ is tangent to PS5-spline $s(x, y)$ at vertex $V_{i}$.

Proof. By the local support of the B-splines and by definition (3.9), we see that

$$
\begin{aligned}
& s\left(V_{i}\right)=\sum_{j=1}^{10} \alpha_{i, j}^{00} c_{i, j}^{v}=T_{i}\left(V_{i}\right), \\
& \frac{\partial}{\partial x} s\left(V_{i}\right)=\sum_{j=1}^{10} \alpha_{i, j}^{10} c_{i, j}^{v}=\frac{\partial}{\partial x} T_{i}\left(V_{i}\right), \\
& \frac{\partial}{\partial y} s\left(V_{i}\right)=\sum_{j=1}^{10} \alpha_{i, j}^{01} c_{i, j}^{v}=\frac{\partial}{\partial y} T_{i}\left(V_{i}\right),
\end{aligned}
$$




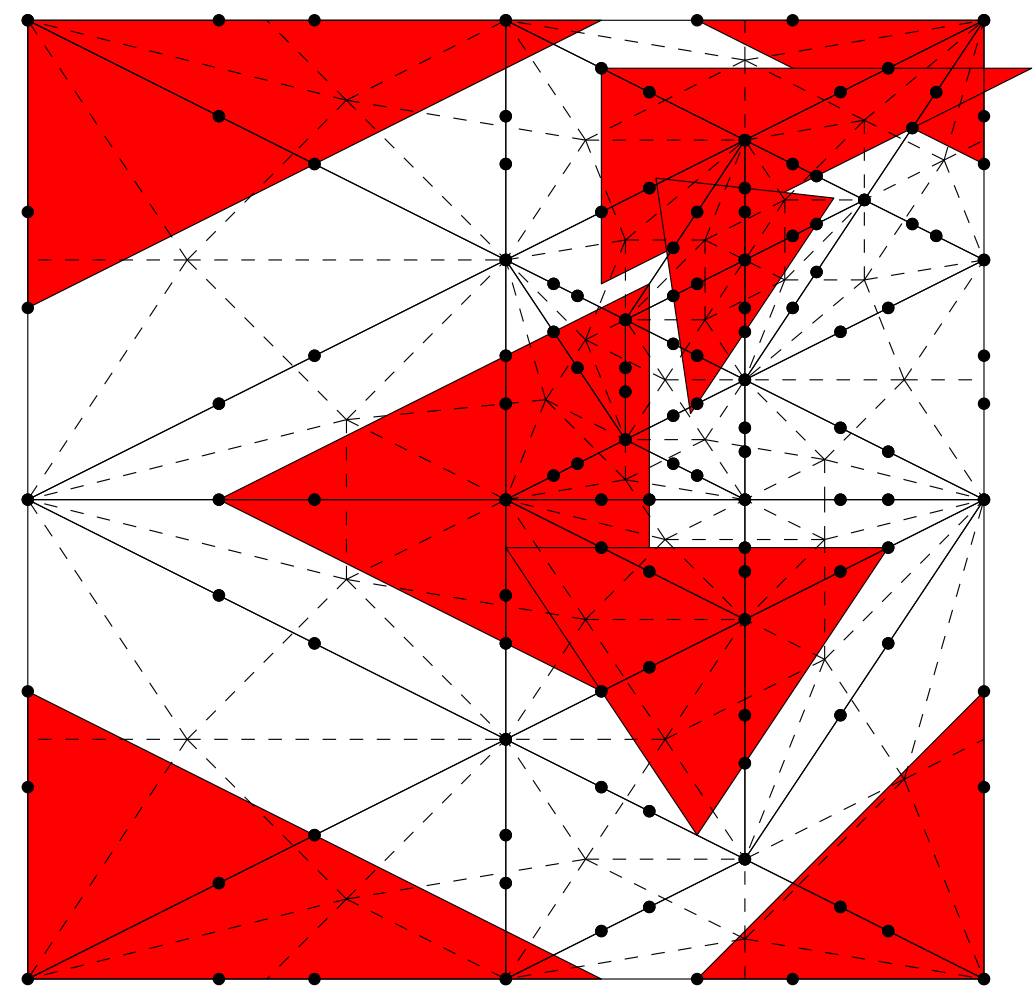

Figure 5: A triangulation with the PS5-points and a few optimal PS5-triangles.

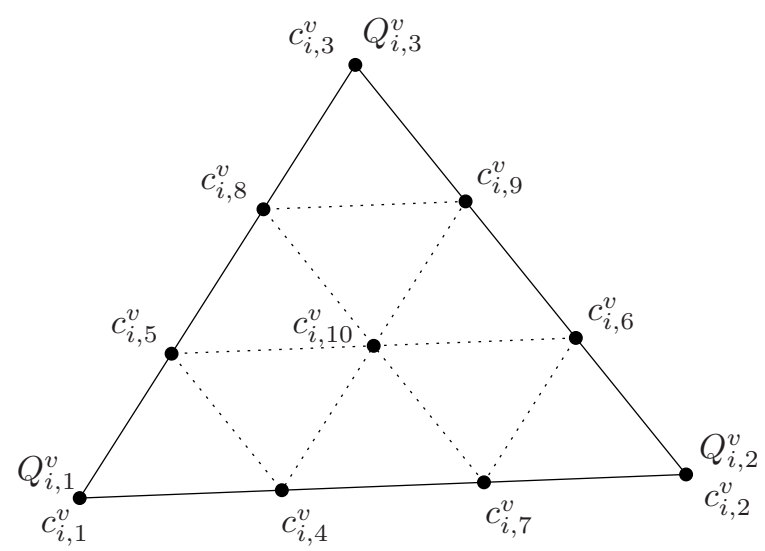

Figure 6: Schematic representation of the B-spline coefficients $c_{i, j}^{v}, j=1, \ldots, 10$, with respect to PS5-triangle $t_{i}\left(Q_{i, 1}^{v}, Q_{i, 2}^{v}, Q_{i, 3}^{v}\right)$.

which shows that $T_{i}(x, y)$ is tangent to the spline at $V_{i}$. 

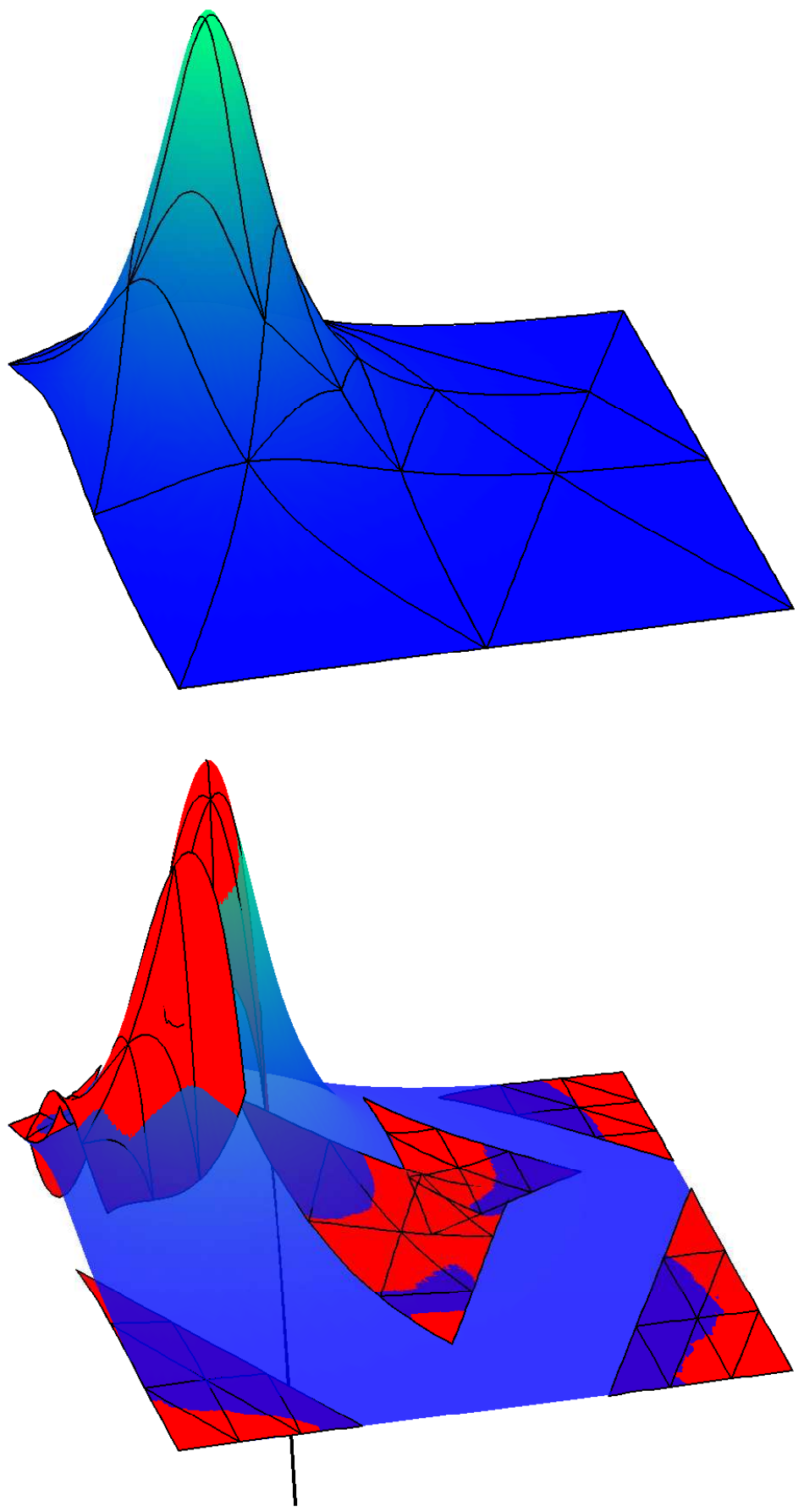

Figure 7: A PS5-spline surface with a few control polynomials corresponding to the triangulation shown in Figure 5. 
Figure 7 shows a PS5-spline surface together with a few control polynomials. The spline is an approximation of the function $f(x, y)=\left(\exp \left((x-0.52)^{2}+(y-0.48)^{2}\right)-0.95\right)^{-1}$ on domain $\Omega=$ $[-1,1] \times[-1,1]$, where the data points are located at the vertices of the triangulation in Figure 5 .

We define control points as

$$
\mathbf{c}_{i, j}^{v}=\left(X_{i, j}^{v}, Y_{i, j}^{v}, c_{i, j}^{v}\right), \quad \mathbf{c}_{k}^{t}=\left(X_{k}^{t}, Y_{k}^{t}, c_{k}^{t}\right),
$$

with $i=1, \ldots, n_{v}, j=1, \ldots, 10$, and $k=1, \ldots, n_{t}$. Here, $c_{i, j}^{v}$ and $c_{k}^{t}$ are the B-spline coefficients in representation (2.9). The points $Q_{i, j}^{v}=\left(X_{i, j}^{v}, Y_{i, j}^{v}\right)$ and $Q_{k}^{t}=\left(X_{k}^{t}, Y_{k}^{t}\right)$ satisfy

$$
\sum_{i=1}^{n_{v}} \sum_{j=1}^{10} X_{i, j}^{v} B_{i, j}^{v}(x, y)+\sum_{k=1}^{n_{t}} X_{k}^{t} B_{k}^{t}(x, y)=x
$$

and

$$
\sum_{i=1}^{n_{v}} \sum_{j=1}^{10} Y_{i, j}^{v} B_{i, j}^{v}(x, y)+\sum_{k=1}^{n_{t}} Y_{k}^{t} B_{k}^{t}(x, y)=y .
$$

Since the PS5-spline basis forms a convex partition of unity, it follows that the graph of spline (2.9) lies inside the convex hull of the control points (4.2).

Property 4.2. In order to satisfy (4.3), the points $Q_{i, 1}^{v}, Q_{i, 2}^{v}$ and $Q_{i, 3}^{v}$ are the three vertices of PS5-triangle $t_{i}$. The remaining $Q_{i, j}^{v}$ are found as

$$
\begin{aligned}
Q_{i, 4}^{v} & =\frac{2}{3} Q_{i, 1}^{v}+\frac{1}{3} Q_{i, 2}^{v}, \quad Q_{i, 5}^{v}=\frac{2}{3} Q_{i, 1}^{v}+\frac{1}{3} Q_{i, 3}^{v}, \quad Q_{i, 6}^{v}=\frac{2}{3} Q_{i, 2}^{v}+\frac{1}{3} Q_{i, 3}^{v}, \\
Q_{i, 7}^{v} & =\frac{2}{3} Q_{i, 2}^{v}+\frac{1}{3} Q_{i, 1}^{v}, \quad Q_{i, 8}^{v}=\frac{2}{3} Q_{i, 3}^{v}+\frac{1}{3} Q_{i, 1}^{v}, \quad Q_{i, 9}^{v}=\frac{2}{3} Q_{i, 3}^{v}+\frac{1}{3} Q_{i, 2}^{v}, \\
Q_{i, 10}^{v} & =\frac{1}{3} Q_{i, 1}^{v}+\frac{1}{3} Q_{i, 2}^{v}+\frac{1}{3} Q_{i, 3}^{v} .
\end{aligned}
$$

The point $Q_{k}^{t}$ is situated inside triangle $\mathcal{T}_{k}\left(V_{1}, V_{2}, V_{3}\right)$, see Figure 2, as

$$
Q_{k}^{t}=\frac{1}{5}\left(V_{1}+V_{2}+V_{3}\right)+\frac{2}{5} Z=\frac{1+2 z_{1}}{5} V_{1}+\frac{1+2 z_{2}}{5} V_{2}+\frac{1+2 z_{3}}{5} V_{3} .
$$

Proof. The polynomial in Bernstein-Bézier representation (2.1), where the Bézier ordinates are taken as the $x$-coordinates (or $y$-coordinates) of the corresponding Bézier domain points, is equal to the plane $z=x$ (or $z=y$ ). Referring to Figure 6 , we obtain that by the choice of $Q_{i, j}^{v}$ and by relation (3.9) at vertex $V_{i}=\left(x_{i}, y_{i}\right)$ it holds

$$
\begin{aligned}
& \sum_{j=1}^{10} \alpha_{i, j}^{00} X_{i, j}^{v}=x_{i}, \quad \sum_{j=1}^{10} \alpha_{i, j}^{00} Y_{i, j}^{v}=y_{i} \\
& \sum_{j=1}^{10} \alpha_{i, j}^{10} X_{i, j}^{v}=1, \quad \sum_{j=1}^{10} \alpha_{i, j}^{10} Y_{i, j}^{v}=0 \\
& \sum_{j=1}^{10} \alpha_{i, j}^{01} X_{i, j}^{v}=0, \quad \sum_{j=1}^{10} \alpha_{i, j}^{01} Y_{i, j}^{v}=1 \\
& \sum_{j=1}^{10} \alpha_{i, j}^{a b} X_{i, j}^{v}=0, \quad \sum_{j=1}^{10} \alpha_{i, j}^{a b} Y_{i, j}^{v}=0, \quad 2 \leq a+b \leq 3 .
\end{aligned}
$$


By the construction of the B-splines in $\S 3.1$ and $\S 3.2$, the value of a PS5-spline at a split point can be computed through a particular cubic polynomial evaluated at the split point. We consider the macro-triangle $\mathcal{T}_{k}\left(V_{1}, V_{2}, V_{3}\right)$ depicted in Figure 2. In case of the two PS5-splines in the left hand sides of equations (4.3), we look at two cubic polynomials $p_{3}^{x}(\tau)$ and $p_{3}^{y}(\tau)$ defined on the triangle with vertices (3.2). The Bézier ordinates of $p_{3}^{x}(\tau)$ are found by using (4.6), i.e.,

$$
\begin{array}{lll}
b_{300}^{x}=\frac{3}{5} x_{1}+\frac{2}{5}\left(z_{1} x_{1}+z_{2} x_{2}+z_{3} x_{3}\right), & b_{210}^{x}=\frac{2}{3} b_{300}^{x}+\frac{1}{3} b_{030}^{x}, & b_{201}^{x}=\frac{2}{3} b_{300}^{x}+\frac{1}{3} b_{003}^{x}, \\
b_{030}^{x}=\frac{3}{5} x_{2}+\frac{2}{5}\left(z_{1} x_{1}+z_{2} x_{2}+z_{3} x_{3}\right), & b_{021}^{x}=\frac{2}{3} b_{030}^{x}+\frac{1}{3} b_{003}^{x}, & b_{120}^{x}=\frac{2}{3} b_{030}^{x}+\frac{1}{3} b_{300}^{x}, \\
b_{003}^{x}=\frac{3}{5} x_{3}+\frac{2}{5}\left(z_{1} x_{1}+z_{2} x_{2}+z_{3} x_{3}\right), & b_{102}^{x}=\frac{2}{3} b_{003}^{x}+\frac{1}{3} b_{300}^{x}, & b_{012}^{x}=\frac{2}{3} b_{003}^{x}+\frac{1}{3} b_{030}^{x},
\end{array}
$$

and by the definition of $Q_{k}^{t}$, i.e.,

$$
b_{111}^{x}=X_{k}^{t}=\frac{1}{3} b_{300}^{x}+\frac{1}{3} b_{030}^{x}+\frac{1}{3} b_{003}^{x} .
$$

It follows that

$$
p_{3}^{x}(\tau)=\tau_{1} b_{300}^{x}+\tau_{2} b_{030}^{x}+\tau_{3} b_{003}^{x} .
$$

We then obtain that the value of $p_{3}^{x}(\tau)$ at split point $Z$ with barycentric coordinates $\left(z_{1}, z_{2}, z_{3}\right)$ equals $\left(z_{1} x_{1}+z_{2} x_{2}+z_{3} x_{3}\right)$. In a similar way, we find that the value of $p_{3}^{y}(\tau)$ evaluated at the same point is equal to $\left(z_{1} y_{1}+z_{2} y_{2}+z_{3} y_{3}\right)$. Thus,

$$
\begin{aligned}
& X_{k}^{t} B_{k}^{t}(Z)+\sum_{i=1}^{3} \sum_{j=1}^{10} X_{i, j}^{v} B_{i, j}^{v}(Z)=z_{1} x_{1}+z_{2} x_{2}+z_{3} x_{3}, \\
& Y_{k}^{t} B_{k}^{t}(Z)+\sum_{i=1}^{3} \sum_{j=1}^{10} Y_{i, j}^{v} B_{i, j}^{v}(Z)=z_{1} y_{1}+z_{2} y_{2}+z_{3} y_{3} .
\end{aligned}
$$

Using interpolation problem (2.8) and (4.6)-(4.7), it follows that the proposed $Q_{i, j}^{v}$ and $Q_{k}^{t}$ satisfy the conditions (4.3).

We note that $\mathbf{c}_{i, j}^{v}, j=1, \ldots, 10$, are the Bézier control points of the control polynomial $T_{i}(x, y)$. They can be organized in a Bézier control net. Figure 8 shows the Bézier control nets of a few control polynomials of the PS5-spline depicted in Figure 7.

These control points can be used to interactively change the shape of the spline surface in a predictable way. Because of the local support of the B-splines, a change of such a control point will only affect the spline patches related to a few triangles in the triangulation.

\subsection{Bézier ordinates of a PS5-spline}

The Bézier ordinates of a PS5-spline in representation (2.9) can be computed in a stable way from its coefficients $c_{i, j}^{v}$ and $c_{k}^{t}$. We consider again the triangle $\mathcal{T}_{k}\left(V_{1}, V_{2}, V_{3}\right)$ in Figure 2. The Bézier ordinates are schematically represented in Figure 9.

Because of the $C^{3}$-smoothness of the PS5-spline at vertex $V_{1}$ and the local support of the B-splines, the Bézier ordinates $d_{1}, \ldots, d_{16}$ in the neighbourhood of $V_{1}$ are completely determined by control polynomial $T_{1}(x, y)$. It has already been shown that the Bézier ordinates of the B-splines $B_{1, j}^{v}(x, y)$, $j=1, \ldots, 10$, in the neighbourhood of vertex $V_{1}$ can be computed as the Bézier ordinates of the Bernstein polynomials of degree three with respect to PS5-triangle $t_{1}$ after subdivision. Hence, 


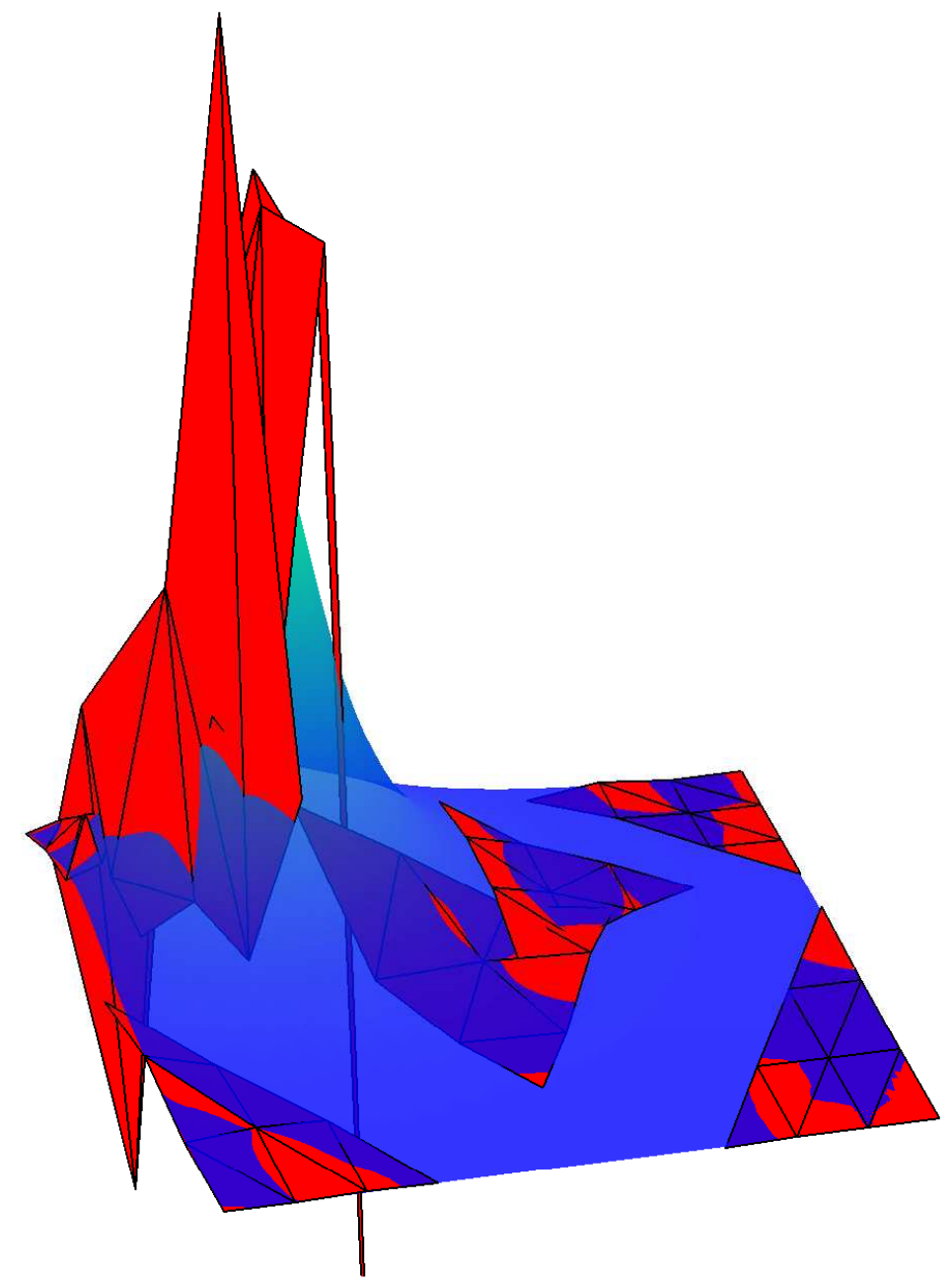

Figure 8: The control Bézier nets of a few control polynomials of the PS5-spline shown in Figure 7.

the ordinates $d_{1}, \ldots, d_{16}$ of the PS5-spline can be simply found by subdividing control polynomial $T_{1}(x, y)$. We compute these ordinates using two stages of subdivision. In the first stage we subdivide the control polynomial onto the triangle with the PS5-points $V_{1}, S_{12}$ and $S_{13}$ as its three vertices. In view of Property 3.3, the barycentric coordinates of these points with respect to PS5-triangle $t_{1}$ are $\left(\gamma_{1,1}, \gamma_{1,2}, \gamma_{1,3}\right),\left(L_{12,1}, L_{12,2}, L_{12,3}\right)$ and $\left(L_{13,1}, L_{13,2}, L_{13,3}\right)$, respectively. We denote the Bézier ordinates of the control polynomial on this finer triangle by $e_{1, j}, j=1, \ldots, 10$, as indicated in Figure 10. They can be computed using only convex combinations through the multi-affine de Casteljau algorithm. The formulae are given for the sake of completeness. Referring to Figure 6, let

$$
\begin{aligned}
& e_{300}=c_{1,1}^{v}, \quad e_{210}=c_{1,4}^{v}, \quad e_{201}=c_{1,5}^{v}, \quad e_{030}=c_{1,2}^{v}, \quad e_{021}=c_{1,6}^{v}, \quad e_{1,120}=c_{1,7}^{v}, \\
& e_{003}=c_{1,3}^{v}, \quad e_{102}=c_{1,8}^{v}, \quad e_{012}=c_{1,9}^{v}, \quad e_{111}=c_{1,10}^{v} .
\end{aligned}
$$




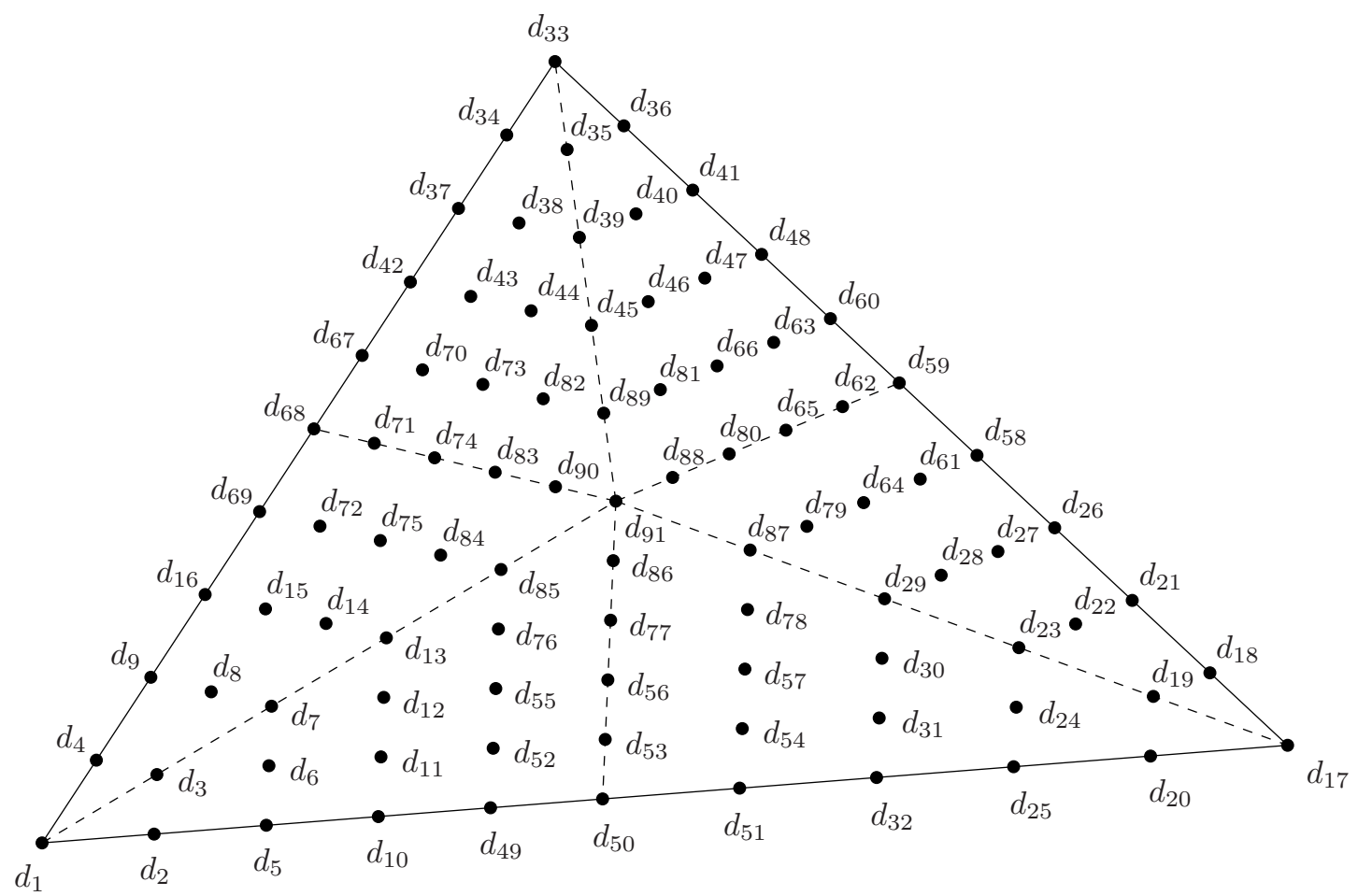

Figure 9: Schematic representation of the Bézier ordinates of a PS5-spline.

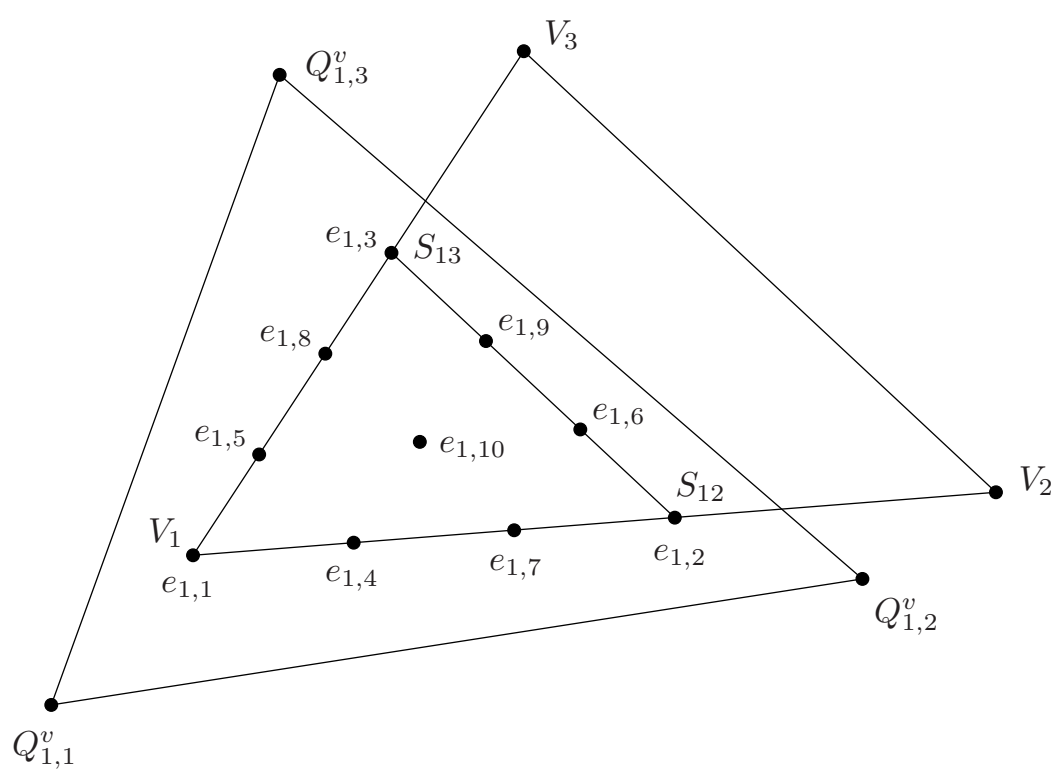

Figure 10: Schematic representation of the Bézier ordinates $e_{1, j}, j=1, \ldots, 10$, of the subdivided control polynomial $T_{1}(x, y)$ onto the triangle with vertices $V_{1}, S_{12}$ and $S_{13}$. 
Applying the de Casteljau algorithm, we get

$$
\begin{aligned}
& e_{1, i j k}=\gamma_{1,1} e_{i+1 j k}+\gamma_{1,2} e_{i j+1 k}+\gamma_{1,3} e_{i j k+1}, \\
& e_{2, i j k}=L_{12,1} e_{i+1 j k}+L_{12,2} e_{i j+1 k}+L_{12,3} e_{i j k+1}, \\
& e_{3, i j k}=L_{13,1} e_{i+1 j k}+L_{13,2} e_{i j+1 k}+L_{13,3} e_{i j k+1},
\end{aligned}
$$

for all $i+j+k=2$, and

$$
\begin{aligned}
& e_{1, i j k}=\gamma_{1,1} e_{1, i+1 j k}+\gamma_{1,2} e_{1, i j+1 k}+\gamma_{1,3} e_{1, i j k+1}, \\
& e_{2, i j k}=L_{12,1} e_{2, i+1 j k}+L_{12,2} e_{2, i j+1 k}+L_{12,3} e_{2, i j k+1}, \\
& e_{3, i j k}=L_{13,1} e_{3, i+1 j k}+L_{13,2} e_{3, i j+1 k}+L_{13,3} e_{3, i j k+1}, \\
& e_{10, i j k}=L_{12,1} e_{1, i+1 j k}+L_{12,2} e_{1, i j+1 k}+L_{12,3} e_{1, i j k+1},
\end{aligned}
$$

for all $i+j+k=1$. Finally,

$$
\begin{aligned}
& e_{1,1}=\gamma_{1,1} e_{1,100}+\gamma_{1,2} e_{1,010}+\gamma_{1,3} e_{1,001} \\
& e_{1,2}=L_{12,1} e_{2,100}+L_{12,2} e_{2,010}+L_{12,3} e_{2,001} \\
& e_{1,3}=L_{13,1} e_{3,100}+L_{13,2} e_{3,010}+L_{13,3} e_{3,001} \\
& e_{1,4}=L_{12,1} e_{1,100}+L_{12,2} e_{1,010}+L_{12,3} e_{1,001} \\
& e_{1,5}=L_{13,1} e_{1,100}+L_{13,2} e_{1,010}+L_{13,3} e_{1,001} \\
& e_{1,6}=L_{13,1} e_{2,100}+L_{13,2} e_{2,010}+L_{13,3} e_{2,001} \\
& e_{1,7}=\gamma_{1,1} e_{2,100}+\gamma_{1,2} e_{2,010}+\gamma_{1,3} e_{2,001} \\
& e_{1,8}=\gamma_{1,1} e_{3,100}+\gamma_{1,2} e_{3,010}+\gamma_{1,3} e_{3,001} \\
& e_{1,9}=L_{12,1} e_{3,100}+L_{12,2} e_{3,010}+L_{12,3} e_{1,001} \\
& e_{1,10}=L_{13,1} e_{10,100}+L_{13,2} e_{10,010}+L_{13,3} e_{10,001}
\end{aligned}
$$

In the second stage we compute the values of $d_{1}, \ldots, d_{16}$ from the ordinates $e_{1, j}, j=1, \ldots, 10$. We use again the multi-affine de Casteljau algorithm. Let

$$
\begin{aligned}
& \tilde{d}_{5}=\lambda_{12} e_{1,4}+\lambda_{21} e_{1,7}, \quad \tilde{\tilde{d}}_{10}=\lambda_{12} e_{1,7}+\lambda_{21} e_{1,2}, \\
& \tilde{d}_{6}=z_{1} e_{1,4}+z_{2} e_{1,7}+z_{3} e_{1,10}, \quad \tilde{\tilde{d}}_{11}=z_{1} e_{1,7}+z_{2} e_{1,2}+z_{3} e_{1,6}, \\
& \tilde{d}_{8}=z_{1} e_{1,5}+z_{2} e_{1,10}+z_{3} e_{1,8}, \quad \tilde{\tilde{d}}_{15}=z_{1} e_{1,8}+z_{2} e_{1,9}+z_{3} e_{1,3}, \\
& \tilde{d}_{10}=\lambda_{12} \tilde{d}_{5}+\lambda_{21} \tilde{d}_{10}, \quad \tilde{d}_{11}=\lambda_{12} \tilde{d}_{6}+\lambda_{21} \tilde{\tilde{d}}_{11}, \\
& \tilde{d}_{13}=z_{1} e_{1,10}+z_{2} e_{1,6}+z_{3} e_{1,9}, \quad \tilde{d}_{12}=z_{1} \tilde{d}_{6}+z_{2} \tilde{\tilde{d}}_{11}+z_{3} \tilde{d}_{13}, \\
& \tilde{d}_{14}=z_{1} \tilde{d}_{8}+z_{2} \tilde{d}_{13}+z_{3} \tilde{\tilde{d}}_{15},
\end{aligned}
$$

then

$$
\begin{aligned}
& d_{1}=e_{1,1}, \quad d_{2}=\lambda_{12} e_{1,1}+\lambda_{21} e_{1,4}, \quad d_{3}=z_{1} e_{1,1}+z_{2} e_{1,4}+z_{3} e_{1,5}, \\
& d_{5}=\lambda_{12} d_{2}+\lambda_{21} \tilde{d}_{5}, \quad d_{10}=\lambda_{12} d_{5}+\lambda_{21} \tilde{d}_{10}, \\
& d_{6}=\lambda_{12} d_{3}+\lambda_{21} \tilde{d}_{6}, \quad d_{11}=\lambda_{12} d_{6}+\lambda_{21} \tilde{d}_{11}, \\
& d_{7}=z_{1} d_{3}+z_{2} \tilde{d}_{6}+z_{3} \tilde{d}_{8}, \quad d_{12}=\lambda_{12} d_{7}+\lambda_{21} \tilde{d}_{12}, \\
& d_{13}=z_{1} d_{7}+z_{2} \tilde{d}_{12}+z_{3} \tilde{d}_{14} .
\end{aligned}
$$

Analogous expressions hold for $d_{4}, d_{8}, d_{9}, d_{14}, d_{15}, d_{16}$. 
Let $e_{2, j}, j=1, \ldots, 10$, be the Bézier ordinates of control polynomial $T_{2}(x, y)$ restricted on the triangle with vertices $\left(V_{2}, S_{23}, S_{21}\right)$, and let $e_{3, j}, j=1, \ldots, 10$, be the Bézier ordinates of control polynomial $T_{3}(x, y)$ restricted on the triangle with vertices $\left(V_{3}, S_{31}, S_{32}\right)$. Using $e_{2, j}$ and $e_{3, j}$, we then obtain the values of $d_{17}, \ldots, d_{48}$ in the neighbourhood of vertices $V_{2}$ and $V_{3}$ in a manner similar to (4.9)-(4.10).

Alternatively, we could compute the values of $d_{1}, \ldots, d_{48}$ using a single stage of subdivision. Later on, in $\S 4.3$, the values of $e_{i, j}, j=1, \ldots, 10$, will also be useful in a different context.

The values of the Bézier ordinates $d_{49}, \ldots, d_{57}$ are obtained from the $C^{3}$-smoothness conditions of the PS5-spline across the edge $Z-R_{12}$. Using

$$
\tilde{d}_{50}=\lambda_{12} \tilde{d}_{10}+\lambda_{21} \tilde{d}_{32}, \quad \tilde{d}_{53}=\lambda_{12} \tilde{d}_{11}+\lambda_{21} \tilde{d}_{31}, \quad \tilde{d}_{56}=\lambda_{12} \tilde{d}_{12}+\lambda_{21} \tilde{d}_{30},
$$

we get

$$
\begin{array}{llll}
d_{49}=\lambda_{12} d_{10}+\lambda_{21} \tilde{d}_{50}, & d_{51}=\lambda_{12} \tilde{d}_{50}+\lambda_{21} d_{32}, & d_{50}=\lambda_{12} d_{49}+\lambda_{21} d_{51}, \\
d_{52}=\lambda_{12} d_{11}+\lambda_{21} \tilde{d}_{53}, & d_{54}=\lambda_{12} \tilde{d}_{53}+\lambda_{21} d_{31}, & d_{53}=\lambda_{12} d_{52}+\lambda_{21} d_{54}, \\
d_{55}=\lambda_{12} d_{12}+\lambda_{21} \tilde{d}_{56}, & d_{57}=\lambda_{12} \tilde{d}_{56}+\lambda_{21} d_{30}, & d_{56}=\lambda_{12} d_{55}+\lambda_{21} d_{57} .
\end{array}
$$

In a similar way we find the values of the Bézier ordinates $d_{58}, \ldots, d_{75}$. Note that the values of $d_{5}, d_{10}, d_{49}, d_{50}, d_{51}, d_{32}$ and $d_{25}$ can be regarded as Bézier ordinates after subdivision of a one-dimensional cubic polynomial with Bézier ordinates $d_{5}, \tilde{d}_{10}, \tilde{d}_{32}$ and $d_{25}$, defined on the interval

$$
\left[\frac{3}{5} V_{1}+\frac{2}{5} R_{12}, \frac{3}{5} V_{2}+\frac{2}{5} R_{12}\right] .
$$

The remaining Bézier ordinates $d_{76}, \ldots, d_{91}$ are obtained as the Bézier ordinates after subdivision of a single cubic polynomial $p_{3}(\tau)$ defined on the triangle with vertices (3.2). Referring to the construction of the B-splines, the polynomial $p_{3}(\tau)$ in Bernstein-Bézier representation (2.1) has as Bézier ordinates

$$
\begin{aligned}
& b_{300}=d_{7}, \quad b_{210}=\tilde{d}_{12}, \quad b_{201}=\tilde{d}_{14}, \quad b_{030}=d_{23}, \quad b_{021}=\tilde{d}_{28}, \quad b_{120}=\tilde{d}_{30}, \\
& b_{003}=d_{39}, \quad b_{102}=\tilde{d}_{44}, \quad b_{012}=\tilde{d}_{46}, \quad b_{111}=c_{k}^{t} .
\end{aligned}
$$

Let

$$
\begin{aligned}
& \tilde{d}_{77}=z_{1} \tilde{d}_{12}+z_{2} \tilde{d}_{30}+z_{3} c_{k}^{t}, \\
& \tilde{d}_{80}=z_{1} c_{k}^{t}+z_{2} \tilde{d}_{28}+z_{3} \tilde{d}_{46}, \\
& \tilde{d}_{83}=z_{1} \tilde{d}_{14}+z_{2} c_{k}^{t}+z_{3} \tilde{d}_{44},
\end{aligned}
$$

we then obtain

$$
\begin{aligned}
& d_{76}=\lambda_{12} d_{13}+\lambda_{21} \tilde{d}_{77}, \quad d_{78}=\lambda_{12} \tilde{d}_{77}+\lambda_{21} d_{29}, \quad d_{77}=\lambda_{12} d_{76}+\lambda_{21} d_{78}, \\
& d_{85}=z_{1} d_{13}+z_{2} \tilde{d}_{77}+z_{3} \tilde{d}_{83}, \quad d_{87}=z_{1} \tilde{d}_{77}+z_{2} d_{29}+z_{3} \tilde{d}_{80}, \quad d_{86}=\lambda_{12} d_{85}+\lambda_{21} d_{87}, \\
& d_{89}=z_{1} \tilde{d}_{83}+z_{2} \tilde{d}_{80}+z_{3} d_{45}, \quad d_{91}=z_{1} d_{85}+z_{2} d_{87}+z_{3} d_{89} .
\end{aligned}
$$

Expressions similar to (4.14) hold for $d_{79}, \ldots, d_{84}, d_{88}$ and $d_{90}$.

Only convex combinations are needed in (4.9)-(4.14) to compute all Bézier ordinates of the PS5spline (2.9) from its spline coefficients. Using the de Casteljau algorithm, the PS5-spline can then be evaluated in a stable way, given its normalized B-spline representation.

More general, if we apply the convex combinations (4.9)-(4.14) to the control points $\mathbf{c}_{i, j}^{v}$ and $\mathbf{c}_{k}^{t}$ in (4.2), we directly get the Bézier control points of the PS5-spline surface. Figure 11 shows the Bézier control net of the PS5-spline depicted in Figure 7. 


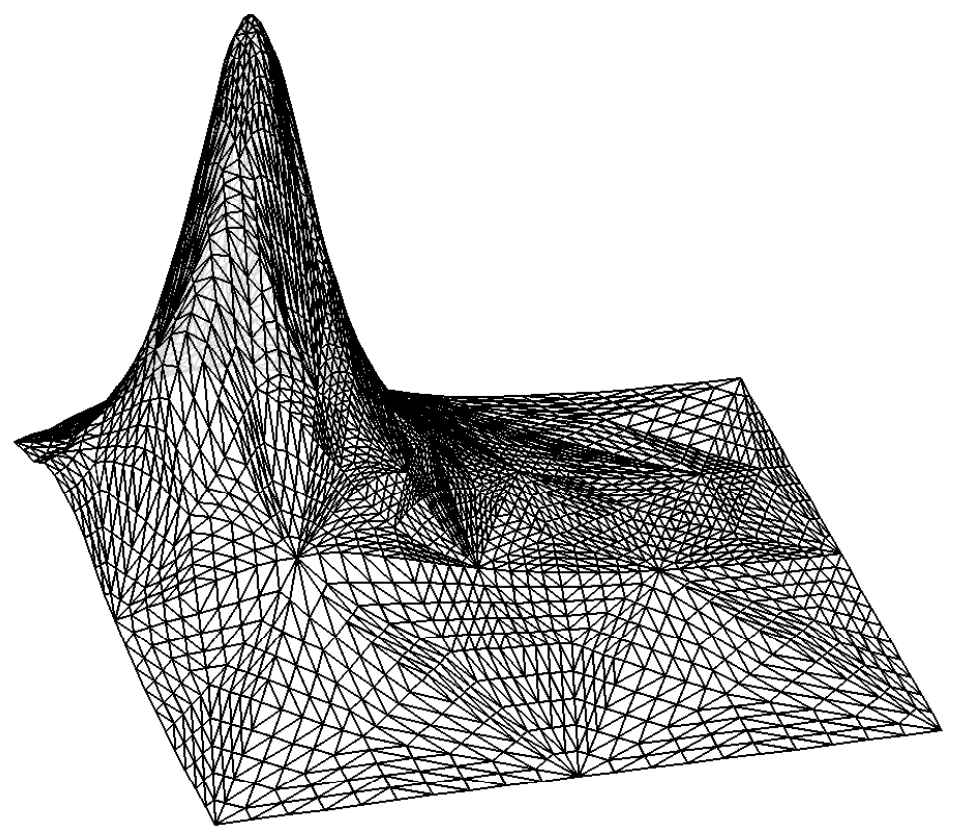

Figure 11: Bézier control net of a PS5-spline surface.

\subsection{Choice of the B-spline coefficients with respect to a triangle}

We now determine specific values for the B-spline coefficients $c_{k}^{t}, k=1, \ldots, n_{t}$, such that the obtained PS5-splines are still able to reproduce quintic polynomials. In this way, their optimal approximation order is retained with less degrees of freedom.

We consider a single triangle $\mathcal{T}_{k}\left(V_{1}, V_{2}, V_{3}\right)$ in $\Delta$, on which we define a quintic polynomial $p_{5}(\tau)$ in Bernstein-Bézier representation (2.1). Its Bézier ordinates are denoted by $\hat{b}_{a b c}, a+b+c=5$. We recall that the control polynomial $T_{1}(x, y)$ of a PS5-spline $s(x, y)$, restricted on the triangle with vertices $\left(V_{1}, S_{12}, S_{13}\right)$, is a cubic polynomial with Bézier ordinates $e_{1, j}, j=1, \ldots, 10$, as defined in $\S 4.2$. By the definition of the control polynomial, the spline $s(x, y)$ has the same function value and derivative values up to order three at vertex $V_{1}$ as the polynomial $p_{5}(\tau)$, if

$$
\begin{array}{ll}
\hat{b}_{500}=e_{1,1}, & \hat{b}_{410}=e_{1,4}, \quad \hat{b}_{401}=e_{1,5}, \\
\hat{b}_{320}=e_{1,7}, & \hat{b}_{311}=e_{1,10}, \quad \hat{b}_{302}=e_{1,8}, \\
\hat{b}_{230}=e_{1,2}, & \hat{b}_{221}=e_{1,6}, \quad \hat{b}_{212}=e_{1,9}, \quad \hat{b}_{203}=e_{1,3} .
\end{array}
$$

We obtain similar conditions to interpolate the function and derivative values at the vertices $V_{2}$ and $V_{3}$.

Referring to Figure 9 , the Bézier ordinates $d_{76}, \ldots, d_{91}$ of a PS5-spline can be computed as the Bézier ordinates after subdivision of a cubic polynomial $p_{3}(\tau)$ defined on the triangle with vertices (3.2). The spline $s(x, y)$ has the same function value and derivative values up to order three at 
split point $Z$ as the polynomial $p_{5}(\tau)$, if the Bézier ordinates $b_{a b c}$ of $p_{3}(\tau)$ satisfy

$$
\begin{aligned}
b_{a b c}= & z_{1}\left(z_{1} \hat{b}_{a+2 b c}+z_{2} \hat{b}_{a+1 b+1 c}+z_{3} \hat{b}_{a+1 b c+1}\right) \\
& +z_{2}\left(z_{1} \hat{b}_{a+1 b+1 c}+z_{2} \hat{b}_{a b+2 c}+z_{3} \hat{b}_{a b+1 c+1}\right) \\
& +z_{3}\left(z_{1} \hat{b}_{a+1 b c+1}+z_{2} \hat{b}_{a b+1 c+1}+z_{3} \hat{b}_{a b c+2}\right),
\end{aligned}
$$

for all $a+b+c=3$. This can be proved as follows. Let $\mathcal{P}_{3}$ and $\mathcal{P}_{5}$ be the blossoms of $p_{3}$ and $p_{5}$, respectively. By the choice (4.15) it can be checked that

$$
\mathcal{P}_{3}\left(\tau^{1}, \tau^{2}, \tau^{3}\right)=\mathcal{P}_{5}\left(\tau^{1}, \tau^{2}, \tau^{3}, z, z\right),
$$

with $z=\left(z_{1}, z_{2}, z_{3}\right)$. The subdivision property of blossoms (see, e.g., (2.5)) completes the proof. Since $c_{k}^{t}$ is equal to $b_{111}$, we get the condition

$$
\begin{aligned}
c_{k}^{t}= & z_{1}\left(z_{1} \hat{b}_{311}+z_{2} \hat{b}_{221}+z_{3} \hat{b}_{212}\right) \\
& +z_{2}\left(z_{1} \hat{b}_{221}+z_{2} \hat{b}_{131}+z_{3} \hat{b}_{122}\right) \\
& +z_{3}\left(z_{1} \hat{b}_{212}+z_{2} \hat{b}_{122}+z_{3} \hat{b}_{113}\right) .
\end{aligned}
$$

Hence, if $c_{k}^{t}$ is chosen as in (4.16), where

$$
\begin{aligned}
& \hat{b}_{311}=e_{1,10}, \quad \hat{b}_{131}=e_{2,10}, \quad \hat{b}_{113}=e_{3,10}, \\
& \hat{b}_{221}=\frac{1}{2}\left(e_{1,6}+e_{2,9}\right), \quad \hat{b}_{122}=\frac{1}{2}\left(e_{2,6}+e_{3,9}\right), \quad \hat{b}_{212}=\frac{1}{2}\left(e_{3,6}+e_{1,9}\right),
\end{aligned}
$$

the corresponding PS5-spline will be able to reproduce quintic polynomials. We can rewrite (4.16)(4.17) to

$$
\begin{aligned}
c_{k}^{t}= & z_{1}\left(z_{1} e_{1,10}+z_{2} e_{1,6}+z_{3} e_{1,9}\right) \\
& +z_{2}\left(z_{1} e_{2,9}+z_{2} e_{2,10}+z_{3} e_{2,6}\right) \\
& +z_{3}\left(z_{1} e_{3,6}+z_{2} e_{3,9}+z_{3} e_{3,10}\right) .
\end{aligned}
$$

Using (4.8) and (4.18), it follows that only convex combinations are needed in the computation of the proposed value of $c_{k}^{t}$ given the other B-spline coefficients $c_{i, j}^{v}$. Thus the graph of such a PS5-spline lies inside the convex hull of the control points $\mathbf{c}_{i, j}^{v}$.

One can model a $C^{2}$-continuous quintic Powell-Sabin spline with the aid of the cubic control polynomials $T_{i}(x, y)$ (or its control points $\mathbf{c}_{i, j}^{v}$ ) and the choice (4.18). For further fine-tuning of the spline, the control points $\mathbf{c}_{k}^{t}$ can then be used.

\section{Concluding remarks}

In this paper a normalized B-spline representation for quintic Powell-Sabin splines was developed. The basis consists of ten basis functions associated with each vertex in the triangulation, and a single basis function associated with each triangle in the triangulation. These basis functions have a local support, they are nonnegative and they form a partition of unity. The construction of the basis functions associated with a vertex relies on the determination of a triangle that must contain a specific set of points. These points only depend on the geometry of the triangulation. The function and derivative values of the basis functions at the vertex are related to the function and derivative values of the cubic Bernstein polynomials defined on that triangle.

The normalized B-spline representation has a number of advantages for computer aided geometric design. It allows us to define control points and cubic control polynomials. The control polynomials 
are tangent to the spline surface at the vertices. They enable a designer to interactively change the shape of the spline in a predictable way. We showed that the Bézier control net of a PS5-spline can be computed from the PS5-control points using only convex combinations. This results in a stable way to evaluate the PS5-spline. We can also benefit from the convex partition of unity in range restricted interpolation. In order to guarantee that a PS5-spline satisfies $L \leq s(x, y) \leq U$ with $L<U$ given constants, it suffices to impose the constraints $L \leq c_{i, j}^{v} \leq U$ and $L \leq c_{k}^{t} \leq \bar{U}$.

Finally, we presented a particular way to determine an appropriate value for the B-spline coefficient associated with a triangle, given the values of the B-spline coefficients associated with the vertices. Only convex combinations are needed in the computation. The corresponding PS5-splines are still able to reproduce quintic polynomials. In [1] another approach was proposed. There, a particular $C^{3}$-smoothness condition is imposed on each triangle in the triangulation. This condition, however, is strongly asymmetric with respect to the vertices of the triangle.

\section{Acknowledgement}

Hendrik Speleers is a Postdoctoral Fellow of the Research Foundation Flanders (Belgium).

\section{References}

[1] P. Alfeld and L.L. Schumaker. Smooth macro-elements based on Powell-Sabin triangle splits. Adv. Comput. Math., 16:29-46, 2002.

[2] P. Dierckx. On calculating normalized Powell-Sabin B-splines. Comput. Aided Geom. Design, 15:61-78, 1997.

[3] P. Dierckx, S. Van Leemput, and T. Vermeire. Algorithms for surface fitting using PowellSabin splines. IMA J. Numer. Anal., 12:271-299, 1992.

[4] G. Farin. Triangular Bernstein-Bézier patches. Comput. Aided Geom. Design, 3:83-127, 1986.

[5] Y. He, M. Jin, X. Gu, and H. Qin. A $C^{1}$ globally interpolatory spline of arbitrary topology. In N. Paragios, O.D. Faugeras, T. Chan, and C. Schnörr, editors, IEEE Workshop on Variational, Geometric and Level Set Methods in Computer Vision, pages 295-306, China, 2005.

[6] M.J. Lai. On $C^{2}$ quintic spline functions over triangulations of Powell-Sabin's type. J. Comput. Appl. Math., 73:135-155, 1996.

[7] M.J. Lai and L.L. Schumaker. Macro-elements and stable local bases for splines on PowellSabin triangulations. Math. Comp., 72:335-354, 2003.

[8] J. Maes and A. Bultheel. Stable multiresolution analysis on triangles for surface compression. Electr. Trans. Numer. Anal., 25:224-258, 2006.

[9] C. Manni and P. Sablonnière. Quadratic spline quasi-interpolants on Powell-Sabin partitions. Adv. Comput. Math., 26:283-304, 2007.

[10] P. Sablonnière. Composite finite elements of class $C^{2}$. In C.K. Chui, L.L. Schumaker, and F.I. Utreras, editors, Topics in Multivariate Approximation, pages 207-217. Academic Press, 1987.

[11] H.P. Seidel. An introduction to polar forms. IEEE Comp. Graph. Appl., 13:38-46, 1993. 
[12] H. Speleers. A normalized basis for reduced Clough-Tocher splines. Technical Report 546, Dept. Comp. Science, K.U. Leuven, 2009.

[13] H. Speleers, P. Dierckx, and S. Vandewalle. Numerical solution of partial differential equations with Powell-Sabin splines. J. Comput. Appl. Math., 189:643-659, 2006.

[14] H. Speleers, P. Dierckx, and S. Vandewalle. Quasi-hierarchical Powell-Sabin B-splines. Comput. Aided Geom. Design, 26:174-191, 2009. 